\title{
Testing the price and affordability of healthy and current (unhealthy) diets and the potential impacts of policy change in Australia
}

\author{
Amanda J. Lee ${ }^{1 *}$, Sarah Kane ${ }^{1}$, Rebecca Ramsey², Elizabeth Good ${ }^{3}$ and Mathew Dick ${ }^{3}$
}

\begin{abstract}
Background: Price and affordability of foods are important determinants of health. Targeted food pricing policies may help improve population diets. However, methods producing comparable data to inform relevant policy decisions are lacking in Australia and globally. The objective was to develop and pilot standardised methods to assess the price, relative price and affordability of healthy (recommended) and current (unhealthy) diets and test impacts of a potential policy change.
\end{abstract}

Methods: Methods followed the optimal approach proposed by INFORMAS using recent Australian dietary intake data and guidelines. Draft healthy and current (unhealthy) diet baskets were developed for five household structures. Food prices were collected in stores in a high and low SES location in Brisbane, Australia. Diet prices were calculated and compared with household incomes, and with potential changes to the Australian Taxation System. Wilcoxen-signed rank tests were used to compare differences in price.

Results: The draft tools and protocols were deemed acceptable at household level, but methods could be refined. All households spend more on current (unhealthy) diets than required to purchase healthy (recommended) diets, with the majority (53-64\%) of the food budget being spent on 'discretionary' choices, including take-away foods and alcohol. A healthy diet presently costs between 20-31\% of disposable income of low income households, but would become unaffordable for these families under proposed changes to expand the GST to apply to all foods in Australia.

Conclusions: Results confirmed that diet pricing methods providing meaningful, comparable data to inform potential fiscal and health policy actions can be developed, but draft tools should be refined. Results suggest that healthy diets can be more affordable than current (unhealthy) diets in Australia, but other factors may be as important as price in determining food choices.

Keywords: INFORMAS, Diet prices, Food prices, Diet affordability, Food affordability, Food policy, Food environments, Healthy diets, Obesity prevention, Non-communicable disease

\footnotetext{
* Correspondence: Amanda.Lee@qut.edu.au

'School of Public Health and Social Work, Queensland University of

Technology, Brisbane, QLD, Australia

Full list of author information is available at the end of the article
} 


\section{Background}

Unhealthy diets are now the major preventable risk factor contributing to the burden of disease in globally and in Australia [1] and are driven by 'obesogenic' food environments affecting food promotion, availability, accessibility and affordability [2]. Data from the Australian Health Survey 2011-12 [3] show that less than seven percent of Australians consume diets consistent with the recommendations of the Australian Dietary Guidelines (ADGs) 2013; [4] at least $35 \%$ of the energy intake of adults and at least $39 \%$ of the energy intake of children are now derived from unhealthy 'discretionary' choices [3], described by the Australian National Health and Medical Research Council as foods and drinks high in saturated fat, added sugar, salt and/or alcohol that are not required for health [4]. Of concern is the contribution of poor diet to the rising rates of overweight and obesity; [4] $25 \%$ of Australian children and $63 \%$ of Australian adults are now overweight or obese [3]. There is an urgent need for nutrition policy actions that can help shift the current intake of the whole population to a healthier diet consistent with dietary recommendations in Australia [4].

\section{Assessing diet price and affordability}

The public perception that healthy foods are expensive is believed to be a key factor contributing to poor dietary choices and diet-related health inequities [4-6]. In developed countries, greater total spending on food tends to be associated with more nutritious dietary patterns [7]. If populations were to follow dietary recommendations, this may lead to higher food costs, with those households with the lowest incomes being most vulnerable, as they spend less per person on food, but a greater proportion of their total expenditure on food [7].

However, as the relative price of 'healthy' and 'unhealthy' foods depends on the unit of measure (i.e., per energy unit, nutrient density, serve or weight) [7], it is not always clear whether 'healthy' foods are really more expensive than 'unhealthy' foods, (e.g., healthy foods such as fruit have a high energy-to-price ratio but can provide specific nutrients at a much lower price than other less healthy foods). Comparisons can be difficult particularly in the context of the total, habitual diet that is the major determinant of diet-related disease $[1,2,4]$. Moreover, as opposed to selected pairs of 'healthy' and 'less healthy' foods, the relative price and affordability of current (unhealthy) and healthy (recommended) diets rarely have been assessed [7].

The International Network for Food and Obesity/noncommunicable diseases (NCDs) Research, Monitoring and Action Support (INFORMAS), a global network of public-interest organisations and researchers that aims to monitor, benchmark and support public and private sector actions to create healthy food environments (i.e., with respect to food composition, labelling, promotion, provision, retailing, price and affordability, and trade and investment) and reduce obesity and non-communicable diseases (NCDs) and their related inequalities [2], has highlighted the need for these data [7]. INFORMAS has identified that, with respect to food price and affordability, the relevant questions which need to be answered to inform public policy are:

- What is the relative price and affordability of 'current' (unhealthy) and 'healthy' (recommended) diets?; and

- What would be the effect of potential policy actions on the relative price and affordability of 'current' (unhealthy) and 'healthy' (recommended) diets? [7]

INFORMAS proposes a step-wise framework, comprising 'minimal', 'expanded' and 'optimal' approaches, to monitor price and affordability of the components of 'healthy' and 'less healthy' diets, that takes into account differences in the available capacity, infrastructure and resources of countries to answer these questions and conduct monitoring activities. The framework is described in detail elsewhere [7].

In Australia, as globally, there are no standardized tools and protocols to assess and compare the price and affordability of healthy (recommended) and current (unhealthy) diets [7]. Various Australian jurisdictions and research groups apply different food baskets as dietary survey instruments, and more than ten different instruments and methods are currently in use [5, 6, 8-16]. None of these accurately reflect current Australian diets [3], nor are entirely consistent with current dietary recommendations $[4,17]$ (with exception of the recently revised Queensland methodology [16]). The 'optimal' approach of the INFORMAS diet price and affordability framework potentially provides the basis to develop a standardized method to assess and compare the price and affordability of healthy (recommended) and current (unhealthy) diets in Australia.

\section{Potential policy actions to improve affordability of healthy diets}

A range of inter-related factors influence food prices, including political, economic, socio-cultural and environmental factors at the local, national and international levels [18, 19]. Food prices may be manipulated by governments through a range of complex policy approaches [19]. Three common pricing strategies to increase the affordability of 'healthy' foods at a state or national level are [7]:

(i) taxes on 'unhealthy' foods e.g., on sugar-sweetened beverages; 
(ii)exemption of 'healthy' foods from goods and service tax (GST) or value added tax; and

(iii)subsidies on 'healthy' foods such as agricultural and transport subsidies, retail price reductions, or voucher systems targeted to high-risk groups.

In Australia, basic healthy foods (such as fruit, vegetables, bread, fresh meat, milk and eggs) are currently exempt from GST, which is applied at a rate of $10 \%$ to all other foods and drinks [20]. The GST status of specific food items in Australia can be determined online at the Australian Taxation Office calculator website [21]. Despite basic healthy foods being exempt from GST in Australia, the limited available (non-comparable) data suggest that the cost of 'healthy' food is higher [5] and has increased more rapidly than the cost of 'unhealthy' food over the last 15 years $[14,16]$. Similar findings have been described internationally $[7,22]$.

As a revenue raising measure, the Australian Government has proposed expanding the GST base by removing exemption of basic healthy foods and/or increasing the rate of GST above $10 \%$ [20]. While the potential effects of these proposed changes on the price of specific, selected foods have been modelled [23, 24], the potential impact on price and affordability of total current and recommended diets has not been determined.

\section{Aim}

This study pilots the development and testing of draft tools and protocols with the potential to be standardized nationally, consistent with the INFORMAS 'optimal' approach [7], to investigate the price, relative price and affordability of healthy (recommended) and current (unhealthy) diets in Australia. It also tests the utility of these methods in determining the effect of potential fiscal policy actions by investigating, as an example, the impacts of the mooted broadening and/or raising of the GST base in Australia on the relative price and affordability of current (unhealthy) and healthy (recommended) diets.

\section{Methods}

\section{Construction of the draft diet basket pricing tools}

Diet baskets were developed for five different household types and structures, in expansion of the one reference household proposed by the INFORMAS approach, to encompass the major options used previously in food price surveys in Australian states and territories and optimize utility: $[6,9,12,14,16]$

- Household 1 (HH1) ( $n=6$ ): adult male 31-50 year old; adult female 31-50 year old; older female 70+ yrs old; boy 14 years old; girl 8 years old; boy 4 years old
- Household $2(\mathrm{HH} 2)(n=3)$ : single parent with 2 children: adult female 31-50 year old; boy 14 years old; girl 8 years old

- Household 3 (HH3) $(n=1)$ : single unemployed person: adult male $31-50$ year old

- Household 4 (HH4) $(n=2)$ : older couple with no children: senior adult male $70+$ yrs old; senior adult female $70+$ yrs old: pensioners

- Household 5 (HH5) $(n=4)$ : adult male 31-50 year old; adult female 31-50 year old; boy 14 years old; girl 8 years old

Development of the current (unhealthy) diet basket followed the INFORMAS optimal approach [7]. It contains foods and quantities based on the results of the dietary component of the Australian Health Survey (AHS) 2011-12 which report major, sub-major and minor food groups by age/gender groups (ABS: data cubes 5.1 and 5.3) [3]. When the draft tool was developed in August 2014, confidential unit record files (CURFs) from the survey had not been released, but it was anticipated that application of the summary data available publically could inform a relatively pragmatic method to estimate current diets, suitable for broader global application under INFORMAS [7]. The current (unhealthy) diet basket is comprised of foods and drinks in the quantities and proportions reported as consumed, for example, with percentage energy from discretionary choices as reported earlier [3] (See Additional file 1: file 2.1).

Ideally, the specific foods included in both diet baskets are culturally acceptable, commonly consumed, widely available, accessible and considered 'every day' rather than luxury items. As the foods and drinks included in the current ('unhealthy') basket reflect actual consumption data, it was presumed that they were deemed by the population as a whole as meeting these requirements.

For the current (unhealthy) diet baskets, the mean amounts of each food sub-group per fortnight were calculated from reported daily intake for each relevant age/ gender group (ABS: Table 5.1 and Table 5.3) [3] and tallied.

Development of the healthy (recommended) diet basket also followed the INFORMAS optimal approach [7] based on the quantitative modelled Foundation Diet recommendations for each age/gender group of the Australian Guide to Healthy Eating which include modelled serve sizes and recommended number of serves [4, 17]. The Foundation diet serve recommendations for household members are shown in Table 1 . The products in the healthy diet basket are analogous to the commonly consumed healthy foods in the current diet, but differ in quantity. The healthy diet basket for each household contains the recommended quantities of foods from the 
Table 1 Foundation diets: recommended numbers of serves per week to comprise the healthy diet baskets

\begin{tabular}{|c|c|c|c|c|c|c|c|c|c|c|c|c|c|c|}
\hline \multirow[b]{3}{*}{$\begin{array}{l}\text { Household } \\
\text { structure }\end{array}$} & \multirow[b]{3}{*}{ Members } & \multicolumn{13}{|c|}{ Omnivore Foundation Diets (modelled serves per week) } \\
\hline & & Starchy veges & $\begin{array}{l}\text { Green \& } \\
\text { brassica } \\
\text { veges }\end{array}$ & $\begin{array}{l}\text { Orange } \\
\text { veges }\end{array}$ & Legumes & $\begin{array}{l}\text { Nuts \& } \\
\text { seeds }\end{array}$ & $\begin{array}{l}\text { Other } \\
\text { veges }\end{array}$ & Fruit & $\begin{array}{l}\text { Wholegrain or higher } \\
\text { fibre cereals/grains }\end{array}$ & $\begin{array}{l}\text { Refined or } \\
\text { lower fibre } \\
\text { cereals/grains }\end{array}$ & $\begin{array}{l}\text { Meats \& alts } \\
\text { (minus red) }\end{array}$ & $\begin{array}{l}\text { Red } \\
\text { meats }\end{array}$ & $\begin{array}{l}\text { Diary foods } 50 \% \\
\text { reduced fat }\end{array}$ & $\begin{array}{l}\text { Poly-unsaturated } \\
\text { margarine }^{a}\end{array}$ \\
\hline & & $75 \mathrm{~g}$ & $75 \mathrm{~g}$ & $75 \mathrm{~g}$ & $75 \mathrm{~g}$ & $30 \mathrm{~g}$ & $75 \mathrm{~g}$ & $150 \mathrm{~g}$ & Equiv $40 \mathrm{~g}$ bread & $\begin{array}{l}\text { Equiv } 40 \mathrm{~g} \\
\text { bread }\end{array}$ & $\begin{array}{l}\text { Equiv } 65 \mathrm{~g} \\
\text { meats }\end{array}$ & $65 \mathrm{~g}$ & Equiv $250 \mathrm{~g}$ milk & $10 \mathrm{~g}$ \\
\hline \multirow[t]{7}{*}{1} & Female $70+$ yrs & 5 & 7 & 7 & 3 & 3 & 14 & 14 & 20 & 8 & 7 & 3 & 28 & 14 \\
\hline & Male $31-50$ year & 7 & 7 & 7 & 7 & 7 & 14 & 14 & 28 & 14 & 7 & 7 & 17 & 28 \\
\hline & Female 31-50 year & 5 & 7 & 7 & 2 & 2 & 14 & 14 & 28 & 14 & 7 & 7 & 17 & 14 \\
\hline & Male 14 years & 7 & 7 & 7 & 2 & 4 & 14 & 14 & 32 & 17 & 7 & 7 & 25 & 14 \\
\hline & Female 8 years & 3.5 & 7 & 7 & 2 & 0 & 10.5 & 10.5 & 19 & 9 & 5.5 & 5 & 11.5 & 5 \\
\hline & Male 4 years & 3.5 & 7 & 7 & 2 & 0 & 10.5 & 10.5 & 19 & 9 & 5.5 & 5 & 14 & 5 \\
\hline & TOTAL & 31 & 42 & 42 & 18 & 16 & 77 & 77 & 146 & 71 & 39 & 34 & 112.5 & 80 \\
\hline \multirow[t]{4}{*}{2} & Female 31-50 year & 5 & 7 & 7 & 2 & 2 & 14 & 14 & 28 & 14 & 7 & 7 & 17 & 14 \\
\hline & Male 14 years & 7 & 7 & 7 & 2 & 4 & 14 & 14 & 32 & 17 & 7 & 7 & 25 & 14 \\
\hline & Female 8 years & 3.5 & 7 & 7 & 2 & 0 & 10.5 & 10.5 & 19 & 9 & 5.55 & 5 & 11.5 & 5 \\
\hline & TOTAL & 15.5 & 21 & 21 & 6 & 6 & 38.5 & 38.5 & 79 & 40 & 19.5 & 19 & 53.5 & 33 \\
\hline \multirow[t]{2}{*}{3} & Male 31-50 year & 7 & 7 & 7 & 7 & 7 & 14 & 14 & 28 & 14 & 7 & 7 & 17 & 28 \\
\hline & TOTAL & 7 & 7 & 7 & 7 & 7 & 14 & 14 & 28 & 14 & 7 & 7 & 17 & 28 \\
\hline \multirow[t]{3}{*}{4} & Female $70+$ yrs & 5 & 7 & 7 & 3 & 3 & 14 & 14 & 20 & 8 & 7 & 3 & 28 & 14 \\
\hline & Male $70+$ yrs & 7 & 7 & 7 & 2 & 4 & 14 & 14 & 28 & 14 & 7 & 7 & 17 & 28 \\
\hline & TOTAL & 12 & 14 & 14 & 5 & 7 & 28 & 28 & 48 & 22 & 14 & 10 & 45 & 42 \\
\hline \multirow[t]{5}{*}{5} & Male 31-50 year & 7 & 7 & 7 & 7 & 7 & 14 & 14 & 28 & 14 & 7 & 7 & 17 & 28 \\
\hline & Female 31-50 year & 5 & 7 & 7 & 2 & 2 & 14 & 14 & 28 & 14 & 7 & 7 & 17 & 14 \\
\hline & Male 14 years & 7 & 7 & 7 & 2 & 4 & 14 & 14 & 32 & 17 & 7 & 7 & 25 & 14 \\
\hline & Female 8 years & 3.5 & 7 & 7 & 2 & 0 & 10.5 & 10.5 & 19 & 9 & 5.5 & 5 & 11.5 & 5 \\
\hline & TOTAL & 22.5 & 28 & 28 & 13 & 13 & 52.5 & 52.5 & 107 & 54 & 26.5 & 26 & 70.5 & 61 \\
\hline
\end{tabular}

Each household allocated $100 \mathrm{~g}$ polyunstructured oil and the rest of the allowance provided as polyunstructured margarine

Serve sizes as specefied in the Australian Dietary Guidelines Educators'Guide at https://www.eatforhealth.gov.au/sites/default/files/files/the_guidelines/n55b_educator_guide_140321.pdf 
five food groups and an allowance for unsaturated oils and spreads, for composite age/gender groups of physical activity level (PAL) 1.4, but, consistent with Australian recommendations [4, 17],does not contain any discretionary choices [4]. As the Foundation Diets were developed for the smallest adults (or in the case of children, the youngest) in each age/gender group, any necessary adjustments were made for height/age; for example, amounts were adjusted by an additional $20 \%$ for the 8 year old girl who was the oldest in her group [17]. Consistent with the modelling [17], the healthy diet basket includes: grain (cereal) foods, in the ratio $2 / 3$ wholegrain and $1 / 3$ refined varieties; cheese, milk, yoghurt and calcium-fortified plant based alternatives, mostly (i.e., $>50 \%$ ) reduced fat, with a maximum of $2-3$ serves of high fat dairy foods (cheese) per week; lean meat (beef, lamb, veal, pork), poultry and plant-based alternatives (with no more than $455 \mathrm{~g}$ red meat per week); a minimum of $140 \mathrm{~g}$ and up to $280 \mathrm{~g}$ fish per week; around 7 eggs per week; a selection of different colours and varieties of vegetables (green and brassica, orange, legumes, starchy veg, other) with a minimum $350 \mathrm{~g}$ per day for adults; a variety of fruit with a minimum of 300 g per day for adults; and an allowance of unsaturated oils or spreads or the nuts/seeds from which they are derived. (See Additional file 1: file 1.1).

For the healthy (recommended) diet baskets, the amounts of each food sub-group (serve size multiplied by recommended number of serves) per fortnight were calculated from omnivore Foundation Diets [17] for each of the specific age/gender groups included in the five household structures and tallied. A variety of fresh, canned, frozen and dried foods was included in the proportions modelled [17] with representative fresh produce being in season for most of the year (e.g., stone fruits in season only in summer were excluded). Luxury products such as imported fruit and vegetables (particularly out of season) and foods with high cost per kilogram (e.g., oysters, smoked salmon) were excluded.

The specific branding of items and product sizes for pricing were not finalized until field testing confirmed that these products were commonly available in pilot stores (See Additional file 1: files 1.2 and 2.2).

\section{Collection of food prices}

One high socio-economic and one low socio-economic Statistical Area Level 2 (SA2) [25] location within $30 \mathrm{~km}$ of Brisbane General Post Office were randomly selected as locations for field testing; these were Indooroopilly (high SES) and Logan (low SES). In both locations, stores of the national supermarket chains available in all state and territories in Australia (Coles, Woolworths and Independent Grocers Australia (IGA)) were included, and the prices of food and drink items in both diet baskets were collected in the second and third weeks of September 2014, including commonly available brands, sizes and 'home-brand' generic items, based on methods previously used in Western Australia [14]. Common 'fast-food'/take-away items (including a Big Mac hamburger from the McDonald's ${ }^{\text {Tx }}$ chain, pizza from the Pizza Hut ${ }^{\mathrm{Tm}}$ chain and fish and chips from independent outlets) and alcoholic beverages (spirits, wine and beer) were priced in the respective take-away and liquor outlets closest to each supermarket in each location.

Data recorded included: usual price as well as the price promotion or sale price; cheapest price for loose (not packaged) fresh produce of that description (e.g., red apples); brand name, and whether the item is branded or generic ('home brand'); and prices of different available sizes.

To finalise the diet baskets, the most commonly available branded items and unit sizes in all six supermarkets were included. Some nutritionally similar products were combined to minimize the number of items in each tool; for example, processed meats such as salami and hot dogs were combined with sausages (See Additional file 1: files 1.2 and 2.2).

No attempt was made to adjust the quantities in the current (unhealthy) diet basket for under-reporting in the AHS 2011-12 [3]. An allowance for edible portion/ as cooked was included in both diet baskets, but not for any post-plate wastage in either basket (Additional file 1: files 1.2 and 2.2).

\section{Performance of draft tools and protocols}

Convergent validity of the constructed healthy (recommended) and current (unhealthy) diet baskets for each age/gender group was assessed by energy and macronutrient analysis using FoodWorks 7 Professional [26] computer program installed with current Australian food composition datasets, and comparing the results with Australian Nutrient Reference Values [27] and nutrient results from the AHS 2011-12 [3] respectively. The food composition database used to analyse the AHS (AUSNUT 2011-13) was not available publically at the time this manuscript was prepared; comparative analysis could be improved by repeating analysis when it is released publically. Price and affordability findings were compared with previous available estimates to determine face validity. Internal validity indicators, such as the ratio of fruit and vegetables between diet baskets, were also assessed and compared.

\section{Diet prices and affordability}

The price of the final 'healthy' (recommended) and current (unhealthy) diet baskets was calculated for each of the five household compositions in each store in each location. 
The potential price increases for all foods in the diet baskets under the mooted changes to the Australian tax system [20] were also determined by application of the information available at the Australian Taxation Office calculator site for businesses [21]. Changes in price were compared to identify potential impacts on diet cost for each household (See Additional file 1: files 1.3, 1.4, 1.5, $2.3,2.4,2.5)$.

Affordability of the healthy (recommended) and current (unhealthy) diet baskets for the average family household was determined by comparing the cost of each diet with the median disposable household income, as per the INFORMAS optimal approach [7]. Median disposable household income derived from recent census data in both locations was transcribed from the Queensland Government Statistician's Office website [28].

For the purposes of calculating the affordability of the healthy (recommended) and current (unhealthy) diet baskets for low income families, indicative minimum disposable income of each household was calculated based on the level of minimum wages and determination of the welfare payments provided by the Department of Human Services (2014) as per the methods used by the Queensland Department of Health [16]. Based on the Queensland Council of Social Services' cost of living research on theoretical low income households [29] assumptions were made for employment, housing type, disability status, savings and investments, child support, education attendance and immunization status of children [16]. Details are included in Table 2.

\section{Data and statistical analysis}

Results were analyzed by a range of metrics, including the current cost of purchasing specific core five food group and discretionary items (including policy relevant items such as alcohol, 'take-away foods' and sugarsweetened beverages) and compared with the component costs of healthy (recommended) diets.

Data were also entered into IBM SPSS statistics Version 21 and Wilcoxen-signed ranks test used to compare the costs of diets between high and low socioeconomic (SES) areas and differences in the cost of diets based on proposed policy changes to GST.

\section{Results}

\section{The diet basket pricing tools}

The daily nutrient analysis of the healthy (recommended) and current (unhealthy) diets for each age/gender group included in the five household structures is presented in Table 3. The foods comprising the diet baskets are presented in Table 4.

As deemed acceptable for modelling outputs to develop the Australian Guide to Healthy Eating [17], the energy content of the constructed healthy (recommended) diet baskets was within $5 \%$ of the Foundation Diet levels and the macronutrient profiles were within the recommended ranges for more than $97 \%$ of values for all age/gender groups [17]. Hence these tools appeared to be valid for use in estimating the cost of healthy diets in this pilot. The energy content of the current (unhealthy) diet baskets was within $5 \%$ of the reported energy intakes of the AHS [3] for the adult male, adult female and the two youngest children, but not for the 14 year old boy or seniors (Table 3). However, at the household level, the mean energy content of the current (unhealthy) diet basket was within $5 \%$ of the reported intakes for all but HH4 (comprising the two seniors) (Table 4). However, as the price findings (Table 5) were consistent with available household expenditure survey data [30] (discussed below) and reflected the ratio of current and recommended intakes for key food groups $[3,4]$, the current (unhealthy) diet basket tools appeared to be valid for use in estimating the cost of current diets in the majority of the households in this pilot study. However results for $\mathrm{HH} 4$ should be applied with caution.

In this pilot study, the diet basket tools were developed when neither detailed confidential unit record data (CURFS) from the AHS 2011-12 [3] nor the food composition database (AUSNUT 2011-13) used in the analysis of that survey, were available publically. Although the draft tools appeared fit for purpose and performed well at face value at household level, differences between analysis of reported and constructed current diets at the individual level suggest that more accurate and specific current (unhealthy) diet basket tools may be able to be produced for Australia by accessing and analysing the AHS 2011-12 CURFS when available [3]. More broadly, it is noted that this more precise approach may be too complex for application globally, as detailed dietary survey data are not easily accessible in all countries and technical capacity to analyse individual records may be limited. Therefore the more pragmatic approach used in this pilot study may be more feasible for INFORMAS to monitor the price differential of healthy and current (unhealthy) diets globally [7].

\section{Diet prices}

The price of the healthy (recommended) and current (unhealthy) diet baskets for each of the five household structures at the two locations is shown in Table 5. The higher variance at the high SES location was mainly due to the higher prices in the IGA store compared to the two major supermarket chains in that area. On average, food item prices tended to be around $3 \%$ higher in the high SES location than the low SES location $(Z=2.49$ $p=0.01)$. Take-away foods were relatively more expensive (approximately $9 \%$ ) in the high SES location than the low SES location $(\mathrm{Z}=4.50, p<0.01)$, but alcoholic 
Table 2 Household structures and associated income

\begin{tabular}{|c|c|c|c|}
\hline & Household 1: Household of 6 & Household 2: Single parent with 2 children & $\begin{array}{l}\text { Household 3: Single } \\
\text { unemployed person }\end{array}$ \\
\hline & $\begin{array}{l}\text { Adult male, adult female, older female, } \\
14 \text { years boy, } 8 \text { years girl, } 4 \text { years boy }\end{array}$ & $\begin{array}{l}\text { Adult female, } 14 \text { years boy, } \\
8 \text { years girl }\end{array}$ & Adult male \\
\hline Assumptions & $\begin{array}{l}\text { - The adult male and female are } \\
\text { partnered parents of the three } \\
\text { dependent children } \\
\text { - Adult male is unemployed } \\
\text { and looking for work } \\
\text { - Adult female is a stay-at-home mum } \\
\text { - Older female receives age pension } \\
\text { - The older children attend school } \\
\text { and are fully immunised } \\
\text { - The youngest child attends kindy } 2 \\
\text { days/week and is fully immunised } \\
\text { - The family does not have savings or } \\
\text { investments } \\
\text { - The family is living in } \\
\text { public housing }\end{array}$ & $\begin{array}{l}\text { - The adult female works on a } \\
\text { casual basis at national } \\
\text { minimum wage ( } \$ 20.30 / \mathrm{h}) \\
\text { for } 25 \mathrm{~h} \text { a week for } 39 \\
\text { weeks per year (not during } \\
\text { school holidays) } \\
\text { - The adult female does not } \\
\text { receive child support from } \\
\text { the children's father } \\
\text { - Both children attend school } \\
\text { and are fully immunised } \\
\text { - None of the family are disabled } \\
\text { - The family does not have } \\
\text { savings or investments } \\
\text { - The family is privately renting } \\
\text { their home at } \$ 332 / \text { week }\end{array}$ & $\begin{array}{l}\text { - Has no paid employment } \\
\text { but is looking for work } \\
\text { - Is not studying/training } \\
\text { - Is not disabled } \\
\text { - Has no dependent children } \\
\text { - Does not have savings } \\
\text { or investments } \\
\text { - Is renting a room in } 3 \\
\text { bedroom house } \\
\text { at } \$ 122 / \text { week }\end{array}$ \\
\hline
\end{tabular}

$\begin{array}{ll}\begin{array}{l}\text { Household 4: Older couple with } \\ \text { no children }\end{array} & \begin{array}{l}\text { Household 5: Two parents } \\ \text { with two children }\end{array}\end{array}$

Older male, older female $\quad$ Adult male, adult female,

14 years boy, 8 years girl

Neither are in paid employment - The adult male works on a - Both receive the full age pension permanent basis at national (maximum rate)

- Neither are disabled

or frail-aged

- The couple has no dependent children

- The couple has some savings earning $\$ 100 /$ fortnight in 'deemed income' (less than asset test for age pension

and as in a term deposit and not easily accessed, not included in fortnightly income)

- The couple is privately renting

their home at $\$ 322 /$ week minimum wage for $38 \mathrm{~h}$ a week $(\$ 16.37 / \mathrm{h})$

- The adult female works on a partime basis at national minimum wage $(\$ 16.37 / \mathrm{h})$ for $6 \mathrm{~h}$ a week - Both children attend school and are fully immunised

- None of the family are disabled - The family has some emergency savings that earn negligible interest - The family is privately renting a 3 bedroom house at \$365/week

INCOME per fortnight

Paid Nil

employment

$\$ 761.25$ (average over year)

Nil

Newstart

$\$ 460.90$ (adult male)

N/A

Parenting

$\$ 460.90$ (adult female)

Payment

Family Tax \$568.40/fortnight and

Benefit A and $\quad \$ 2,179.05 /$ year

supplement $\quad$ (\$83.80/fortnight)

Family Tax \$146.44/fortnight

Benefit B and and \$354.04/year

supplement (\$13.61/fortnight)

Age Pension $\quad \$ 766.00$ (older female)

Age Pension $\quad \$ 62.90$ (older female)

Supplement

$\$ 43.26$

Supplement

Rent

N/A (as live in public housing)

Assistance

Income

$\$ 359.60 /$ year (\$13.83/fortnight)

Support Bonus (adult male and female)

N/A (as youngest child is not under 8 years)

$\$ 396.20 /$ fortnight and \$1.452.70/ year ( $\$ 55.87 /$ fortnight)

$\$ 102.20 /$ fortnight and \$354.04/year (\$13.61/fortnight)

N/A

N/A

$\$ 9.69$

$\$ 147.98$

N/A
$\$ 510.50$ (receives maximum) N/A

N/A

N/A

N/A

N/A

N/A

N/A

N/A

$\$ 8.70$

$\$ 84.27$

\$215.60/year (\$8.29/

fortnight)

$\$ 1244.12$ (adult male) $\$ 196.44$ (adult female)

N/A

N/A (as youngest child is not under 8 years)

\$396.20/fortnight and $\$ 1,452.70 /$ year (\$55.87/fortnight)

$\$ 102.20 /$ fortnight and \$354.04/year (\$13.61/fortnight)

$\$ 1,154.80$ (couple payment) N N/A

N/A

$\$ 9.69$

$\$ 147.98$ 
Table 2 Household structures and associated income (Continued)

\begin{tabular}{|c|c|c|c|c|c|}
\hline $\begin{array}{l}\text { Low Income } \\
\text { Supplement }\end{array}$ & N/A & N/A & N/A & N/A & N/A \\
\hline $\begin{array}{l}\text { Low Income } \\
\text { Family } \\
\text { Supplement }\end{array}$ & N/A & $\$ 300 /$ year (\$11.53/fortnight) & N/A & N/A & $\$ 300 /$ year (\$11.53/fortnight) \\
\hline $\begin{array}{l}\text { Single Income } \\
\text { Family } \\
\text { Supplement }\end{array}$ & N/A & N/A & N/A & N/A & N/A \\
\hline $\begin{array}{l}\text { Large Family } \\
\text { Supplement }\end{array}$ & $\$ 12.04$ & N/A & N/A & N/A & N/A \\
\hline $\begin{array}{l}\text { School Kid } \\
\text { Bonus }\end{array}$ & $\$ 1,230 /$ year (\$47.30/fortnight) & $\$ 1,230 /$ year ( $\$ 47.30 /$ fortnight) & N/A & N/A & $\begin{array}{l}\$ 1,230 / \text { year }(\$ 47.30 / \\
\text { fortnight) }\end{array}$ \\
\hline $\begin{array}{l}\text { Childcare } \\
\text { benefit }\end{array}$ & Paid directly to kindergarten & N/A & N/A & N/A & N/A \\
\hline $\begin{array}{l}\text { Childcare } \\
\text { rebate }\end{array}$ & Paid directly to kindergarten & N/A & N/A & N/A & N/A \\
\hline $\begin{array}{l}\text { INCOME TAX } \\
\text { PAID }\end{array}$ & Nil & $\begin{array}{l}\text { Nil due to low income tax offset ( } \$ 302 \text { withheld } \\
\text { from wages is refunded with tax return) }\end{array}$ & Nil & Nil & $\begin{array}{l}\$ 2,278 / \text { year ( } \$ 87.62 / \\
\text { fortnight) (after allowing for } \\
\text { low income tax offset) }\end{array}$ \\
\hline TOTAL & $\$ 2,679.38 /$ fortnight & $\$ 1,545.63 /$ fortnight & $\$ 611.76 /$ fortnight & $\$ 1,389.40 /$ fortnight & $\$ 2,137.32 /$ fortnight \\
\hline
\end{tabular}

Source: Based on Queensland Department of Health 2015 (16)

1) Government payments based on payments and rates available April 2014 at: www.humanservices.gov.au

2) Salaries calculated on national minimum wage at www.fairwork.gov.au $\$ 17.29 / \mathrm{h}$, casual add $25 \%$ loading to $\$ 21.61 / \mathrm{h}$

3) Work status based on 2011 Census data for Queensland (couple households tend to have a fulltime working male and a part-time working female; or when the male is looking for work the female is typically also

out-of-work; for single-parent households, more females tend to work part-time until children are over 14 years)

4) Average rent varies by location (SA2): http://www.abs.gov.au/ausstats/abs@.nsf/Lookup/by\%20Subject/4130.0 2013-14 Main\%20Features Housing\%20Costs\%20and\%20Affordability 5 Estimated rent based on

average of median rates across Queensland postcodes for March 2014 quarter from the Rental Tenancy Authority

5) As all households exceeded maximum fortnightly rent, maximum rent assistance was paid

6) Immunisation status and child support assumed to be able to receive the maximum Government payments 
Table 3 Energy and macronutrient analysis of constructed healthy (recommended) [3] and current (unhealthy) diets [4] for select age/gender groups used to develop the household diet baskets

\begin{tabular}{|c|c|c|c|c|c|c|c|}
\hline \multirow{2}{*}{$\begin{array}{l}\text { Age and } \\
\text { gender group }\end{array}$} & \multirow[t]{2}{*}{ Diet } & \multicolumn{6}{|l|}{ Dietary Analysis } \\
\hline & & $\begin{array}{l}\text { Energy intake } \\
\text { (kJ/day) }\end{array}$ & $\begin{array}{l}\% \text { Energy } \\
\text { from protein }\end{array}$ & $\begin{array}{l}\% \text { Energy } \\
\text { from total fat }\end{array}$ & $\begin{array}{l}\text { \% Energy from } \\
\text { saturated fat }\end{array}$ & $\begin{array}{l}\text { \% Energy from } \\
\text { carbohyd-rate }\end{array}$ & $\begin{array}{l}\% \text { Energy } \\
\text { from alcohol }\end{array}$ \\
\hline \multirow{4}{*}{$\begin{array}{l}\text { Adult male } \\
19-50 \text { year }\end{array}$} & Constructed current (unhealthy) diet ${ }^{a}$ & 10670 & 17 & 32 & 12 & 47 & 4 \\
\hline & Mean reported intake (AHS) [1] & 10220 & 18 & 32 & 12 & 43 & 6 \\
\hline & Constructed healthy diet ${ }^{\mathrm{a}}$ & 8750 & 22 & 33 & 9 & 45 & 0 \\
\hline & $\begin{array}{l}\text { Recommended Foundation diet } \\
(\mathrm{Ht} 1.6 \mathrm{~m} \text { PAL 1.4) [2] }\end{array}$ & 9000 & $15-25$ & $20-35$ & $<10$ & $45-65$ & $<5$ \\
\hline \multirow{4}{*}{$\begin{array}{l}\text { Adult female } \\
19-50 \text { year }\end{array}$} & Constructed unhealthy (current) diet ${ }^{a}$ & 7820 & 17 & 30 & 12 & 49 & 4 \\
\hline & Mean reported intake (AHS) [1] & 7540 & 18 & 33 & 12 & 44 & 4 \\
\hline & Constructed healthy diet ${ }^{a}$ & 7370 & 24 & 29 & 9 & 47 & 0 \\
\hline & $\begin{array}{l}\text { Recommended Foundation diet } \\
\text { (Ht } 1.5 \text { m PAL 1.4) [2] }\end{array}$ & 7100 & $15-25$ & $20-35$ & $<10$ & $45-65$ & $<5$ \\
\hline \multirow{4}{*}{$\begin{array}{l}\text { Senior male } \geq \\
70 \text { year }\end{array}$} & Constructed current (unhealthy) diet ${ }^{\mathrm{a}}$ & 9040 & 16 & 30 & 12 & 48 & 4 \\
\hline & Mean reported intake (AHS) [1] & 8170 & 17 & 31 & 12 & 45 & 5 \\
\hline & Constructed healthy diet [2] & 7460 & 26 & 30 & 11 & 43 & 0 \\
\hline & $\begin{array}{l}\text { Recommended Foundation diet }(\mathrm{Ht} \\
1.6 \text { m PAL 1.4) [2] }\end{array}$ & 7300 & $15-25$ & $20 U-35$ & $<10$ & $45-65$ & $<5$ \\
\hline \multirow{4}{*}{$\begin{array}{l}\text { Senior female } \geq \\
70 \text { year }\end{array}$} & Constructed current (unhealthy) diet ${ }^{a}$ & 7200 & 16 & 30 & 12 & 50 & 3 \\
\hline & Mean reported intake (AHS) [1] & 6570 & 18 & 32 & 12 & 44 & 4 \\
\hline & Constructed healthy diet ${ }^{a}$ & 6710 & 25 & 32 & 12 & 44 & 0 \\
\hline & $\begin{array}{l}\text { Recommended Foundation diet } \\
\text { (Ht } 1.5 \text { m PAL 1.4) [2] }\end{array}$ & 6500 & $15-25$ & $20-35$ & $<10$ & $45-65$ & $<5$ \\
\hline \multirow[t]{4}{*}{ Boy 14 years } & Constructed current (unhealthy) diet ${ }^{a}$ & 9130 & 16 & 29 & 12 & 54 & 0 \\
\hline & Mean reported intake (AHS) [1] & 10190 & 17 & 33 & 13 & 49 & 0 \\
\hline & Constructed healthy diet ${ }^{\mathrm{a}}$ & 8770 & 23 & 28 & 9 & 47 & 0 \\
\hline & $\begin{array}{l}\text { Recommended Foundation diet } \\
\text { (PAL 1.4) [2] }\end{array}$ & 9300 & $15-25$ & $20-35$ & $<10$ & $45-65$ & 0 \\
\hline \multirow[t]{4}{*}{ Girl 8 years } & Constructed current (unhealthy) diet ${ }^{a}$ & 6420 & 15 & 27 & 12 & 56 & 0 \\
\hline & Mean reported intake (AHS) [1] & 6430 & 15 & 31 & 13 & 52 & 0 \\
\hline & Constructed healthy diet [3] ${ }^{a}$ & 5890 & 26 & 23 & 9 & 51 & 0 \\
\hline & $\begin{array}{l}\text { Recommended Foundation diet (PAL } \\
\text { 1.4) [2] }\end{array}$ & 6000 & $15-25$ & $20-35$ & $<10$ & $45-65$ & 0 \\
\hline \multirow[t]{4}{*}{ Boy 4 years } & Constructed current (unhealthy) diet ${ }^{a}$ & 7650 & 15 & 30 & 12 & 54 & 0 \\
\hline & Mean reported intake (AHS) [1] & 7640 & 15 & 32 & 14 & 51 & 0 \\
\hline & Constructed healthy diet ${ }^{a}$ & 5110 & 25 & 24 & 10 & 50 & 0 \\
\hline & $\begin{array}{l}\text { Recommended Foundation diet } \\
\text { (PAL 1.4) [2] }\end{array}$ & 5200 & $15-25$ & $20-35$ & $<10$ & $45-65$ & 0 \\
\hline
\end{tabular}

${ }^{\mathrm{a}}$ Analysed in FoodWorks ${ }^{\mathrm{TM}} 7$ Professional using NUTTAB 2010 and AusBrands 2012 data bases

drinks and sugar-sweetened beverages were priced similarly in both locations (Table 5).

All five household structures spend more purchasing current (unhealthy) diets than the amount required to purchase healthy (recommended) diets (Table 5). At both locations, a healthy diet cost $88-99 \%$ of the money currently being spent on food and drinks in households containing children (HH1, HH2 and HH5). Relatively, a healthy diet costs even less than the current (unhealthy) diet in households without children; $84 \%$ in the household of two elderly adults ( $\mathrm{HH} 4)$ and only $66 \%$ in the household consisting of a single male (HH3).

Only $10-15 \%$ of the food dollar is currently being spent on fruit and vegetables, compared to approximately $29 \%$ required to achieve recommended intakes of these important foods. Similarly, less than half of the required amount is being spent on: grain (cereal) foods, particularly wholegrains $(6-8 \%$ currently compared to 
Table 4 Foods comprising healthy (recommended) \& current (unhealthy) diet baskets, five household (HH) structures

\begin{tabular}{|c|c|c|c|c|c|}
\hline \multicolumn{6}{|l|}{ Current (unhealthy) diet basket } \\
\hline & $\mathrm{HH} 1^{a}$ & $\mathrm{HH} 2^{\mathrm{b}}$ & $\mathrm{HH} 3^{\mathrm{c}}$ & $\mathrm{HH} 4^{\mathrm{d}}$ & $\mathrm{HH} 5^{\mathrm{e}}$ \\
\hline Constructed diets: Total energy per HH per day (kJ) & 48890 & 23370 & 10670 & 16240 & 34040 \\
\hline Reported diets: Total energy per HH per day (kJ) & 48590 & 24160 & 10220 & 14740 & 34380 \\
\hline Food & \multicolumn{5}{|c|}{ Total amount per fortnight } \\
\hline \multicolumn{6}{|l|}{ Fruit } \\
\hline Apples, loose (g) & 3100 & 1700 & 500 & 1100 & 2200 \\
\hline Bananas, loose (g) & 3200 & 1700 & 500 & 1200 & 2200 \\
\hline Oranges, loose (g) & 3200 & 1700 & 500 & 1200 & 2200 \\
\hline Fruit salad, canned in juice (g) & 3100 & 1700 & 500 & 1100 & 2200 \\
\hline \multicolumn{6}{|l|}{ Vegetables } \\
\hline Potato, loose (g) & 2100 & 1050 & 400 & 800 & 1450 \\
\hline Sweetcorn, canned (g) & 510 & 240 & 100 & 200 & 340 \\
\hline Broccoli, loose (g) & 300 & 130 & 70 & 140 & 200 \\
\hline White cabbage, loose (g) & 300 & 130 & 70 & 140 & 200 \\
\hline Iceberg lettuce, whole (g) & 540 & 260 & 100 & 200 & 360 \\
\hline Carrot, loose (g) & 760 & 340 & 160 & 320 & 500 \\
\hline Pumpkin (g) & 1100 & 500 & 200 & 500 & 700 \\
\hline Four bean mix, canned (g) & 750 & 350 & 150 & 300 & 500 \\
\hline Diced tomatoes, canned (g) & 1600 & 650 & 400 & 800 & 1050 \\
\hline Onion, loose (g) & 540 & 260 & 100 & 200 & 360 \\
\hline Tomatoes, loose (g) & 900 & 400 & 200 & 400 & 600 \\
\hline Frozen mixed vegetables, pre-packaged (g) & 900 & 400 & 200 & 400 & 600 \\
\hline Frozen peas, pre-packaged (g) & 700 & 300 & 200 & 300 & 500 \\
\hline Baked beans, canned (g) & 825 & 475 & 125 & 250 & 600 \\
\hline \multicolumn{6}{|l|}{ Grain (cereal) foods } \\
\hline Weetbix & 1400 & 700 & 250 & 450 & 950 \\
\hline Wholemeal bread, pre-packaged (g) & 700 & 350 & 150 & 250 & 500 \\
\hline Rolled oats (g) & 600 & 300 & 100 & 200 & 400 \\
\hline White bread, pre-packaged (g) & 4600 & 2200 & 950 & 1550 & 3150 \\
\hline Cornflakes (g) & 1400 & 700 & 250 & 450 & 950 \\
\hline White pasta (g) & 1650 & 800 & 300 & 550 & 1100 \\
\hline White rice (g) & 1650 & 800 & 300 & 550 & 1100 \\
\hline \multicolumn{6}{|l|}{ Meats, poultry, fish, eggs, nuts and seeds } \\
\hline Beef mince (g) & 800 & 375 & 200 & 300 & 575 \\
\hline Lamb loin chops (g) & 790 & 370 & 200 & 300 & 570 \\
\hline Beef rump steak (g) & 840 & 420 & 200 & 300 & 620 \\
\hline Tuna, canned in springwater (g) & 1145 & 500 & 285 & 560 & 785 \\
\hline Chicken breast (g) & 1750 & 730 & 460 & 640 & 1190 \\
\hline Eggs (g) & 1140 & 565 & 240 & 460 & 805 \\
\hline Canned meat and vegetable casserole (g) & 2350 & 1200 & 600 & 650 & 1800 \\
\hline \multicolumn{6}{|l|}{ Milk, yoghurt, cheese and alternatives } \\
\hline Cheddar cheese, full fat (g) & 720 & 370 & 120 & 200 & 490 \\
\hline Milk, full fat (ml) & 9850 & 4850 & 1600 & 2900 & 6450 \\
\hline Cheddar cheese, reduced fat (g) & 310 & 160 & 55 & 80 & 215 \\
\hline
\end{tabular}


Table 4 Foods comprising healthy (recommended) \& current (unhealthy) diet baskets, five household (HH) structures (Continued)

\begin{tabular}{|c|c|c|c|c|c|}
\hline Milk, reduced fat $(\mathrm{ml})$ & 6350 & 3150 & 1000 & 1900 & 4150 \\
\hline Yoghurt, reduced fat (g) & 910 & 440 & 130 & 220 & 570 \\
\hline Yoghurt, flavoured reduced fat (g) & 150 & 0 & 150 & 100 & 150 \\
\hline \multicolumn{6}{|l|}{ Unsaturated oils and spreads } \\
\hline Canola margarine (g) & 120 & 50 & 25 & 65 & 75 \\
\hline Sunflower oil (ml) & 120 & 50 & 25 & 65 & 75 \\
\hline \multicolumn{6}{|l|}{ Discretionary choices } \\
\hline Beer, full strength (ml) & 7235 & 2639 & 3569 & 2986 & 6208 \\
\hline White wine, sparkling (ml) & 1454 & 482 & 498 & 1014 & 980 \\
\hline Whisky (ml) & 163 & 51 & 71 & 107 & 122 \\
\hline Red wine $(\mathrm{ml})$ & 1454 & 482 & 498 & 1014 & 980 \\
\hline Butter (g) & 364 & 181 & 58 & 163 & 239 \\
\hline Muffin, commercial (g) & 3485 & 1521 & 747 & 1217 & 2268 \\
\hline Cream-filled sweet biscuit, pre-packaged (g) & 933 & 320 & 332 & 405 & 652 \\
\hline Muesli bar, pre-packaged (g) & 528 & 322 & 83 & 81 & 405 \\
\hline Peanuts, salted (g) & 295 & 108 & 83 & 137 & 191 \\
\hline Pizza, commercial (g) & 3013 & 1610 & 830 & 573 & 2440 \\
\hline Savoury flavoured biscuits (g) & 1298 & 642 & 249 & 407 & 891 \\
\hline Confectionary (g) & 662 & 342 & 116 & 146 & 458 \\
\hline Chocolate (g) & 321 & 169 & 58 & 61 & 227 \\
\hline Coca Cola (ml) & 14117 & 7468 & 4026 & 2284 & 11493 \\
\hline Meat pie, commercial (g) & 1209 & 632 & 332 & 245 & 964 \\
\hline Frozen lasagne, pre-packaged (g) & 1381 & 767 & 332 & 490 & 1099 \\
\hline Hamburger, commercial (g) & 1306 & 609 & 332 & 324 & 941 \\
\hline Beef sausages (g) & 1557 & 486 & 664 & 822 & 1150 \\
\hline Ham (g) & 1059 & 403 & 332 & 490 & 735 \\
\hline Potato crisps, pre-packaged (g) & 673 & 367 & 71 & 44 & 438 \\
\hline Potato chips, commercial (g) & 1176 & 565 & 249 & 486 & 814 \\
\hline Ice cream (g) & 1948 & 806 & 332 & 727 & 1138 \\
\hline White sugar (g) & 1492 & 724 & 208 & 528 & 931 \\
\hline Salad dressing (ml) & 616 & 305 & 133 & 153 & 437 \\
\hline Tomato sauce (ml) & 641 & 305 & 133 & 153 & 437 \\
\hline Chicken soup, canned (g) & 2714 & 1078 & 598 & 1753 & 1675 \\
\hline Orange fruit drink (ml) & 8201 & 4485 & 1245 & 1861 & 5730 \\
\hline Fish fillet crumbed, pre-packaged (g) & 535 & 266 & 108 & 211 & 374 \\
\hline \multicolumn{6}{|l|}{ Healthy (recommended) diet basket } \\
\hline & $\mathrm{HH} 1^{\mathrm{a}}$ & $\mathrm{HH} 2^{\mathrm{b}}$ & $\mathrm{HH} 3^{\mathrm{c}}$ & $\mathrm{HH} 4^{d}$ & $\mathrm{HH} 5^{\mathrm{C}}$ \\
\hline Constructed diets: Total energy per HH per day (kJ) & 42600 & 22030 & 8750 & 14170 & 30780 \\
\hline Recommended diets: Total energy per HH per day $(\mathrm{kJ})$ & 43100 & 22400 & 9000 & 13800 & 31400 \\
\hline Food & \multicolumn{5}{|c|}{ Total amount per fortnight } \\
\hline \multicolumn{6}{|l|}{ Fruit } \\
\hline Apples, loose (g) & 7910 & 4060 & 1400 & 2800 & 5460 \\
\hline Bananas, loose (g) & 7910 & 4060 & 1400 & 2800 & 5460 \\
\hline Oranges, loose (g) & 7910 & 4060 & 1400 & 2800 & 5460 \\
\hline
\end{tabular}

Vegetables 
Table 4 Foods comprising healthy (recommended) \& current (unhealthy) diet baskets, five household (HH) structures (Continued)

\begin{tabular}{|c|c|c|c|c|c|}
\hline Potato, loose (g) & 2970 & 1620 & 700 & 800 & 2320 \\
\hline Sweetcorn, canned (g) & 1485 & 810 & 350 & 400 & 1160 \\
\hline Broccoli, loose (g) & 2170 & 1120 & 350 & 700 & 1470 \\
\hline White cabbage, loose (g) & 2170 & 1120 & 350 & 700 & 1470 \\
\hline Iceberg lettuce, whole (g) & 2170 & 1120 & 350 & 700 & 1470 \\
\hline Carrot, loose (g) & 3255 & 1680 & 525 & 1050 & 2205 \\
\hline Pumpkin (g) & 3255 & 1680 & 525 & 1050 & 2205 \\
\hline Four bean mix, canned (g) & 2760 & 960 & 1050 & 750 & 2010 \\
\hline Diced tomatoes, canned (g) & 2373 & 1218 & 420 & 840 & 1638 \\
\hline Onion, loose (g) & 2373 & 1218 & 420 & 840 & 1638 \\
\hline Tomatoes, loose (g) & 2373 & 1218 & 420 & 840 & 1638 \\
\hline Frozen mixed vegetables, pre-packaged (g) & 2373 & 1218 & 420 & 840 & 1638 \\
\hline Frozen peas, pre-packaged (g) & 2373 & 1218 & 420 & 840 & 1638 \\
\hline \multicolumn{6}{|l|}{ Grain (cereal) foods } \\
\hline Weetbix & 2896 & 1656 & 560 & 720 & 2216 \\
\hline Wholemeal bread, pre-packaged (g) & 5792 & 3312 & 1120 & 1440 & 4432 \\
\hline Rolled oats (g) & 8688 & 4968 & 1680 & 2160 & 6648 \\
\hline White bread, pre-packaged (g) & 1133 & 669 & 224 & 256 & 893 \\
\hline Cornflakes (g) & 850 & 502 & 168 & 192 & 670 \\
\hline White pasta (g) & 2124 & 1254 & 420 & 480 & 1674 \\
\hline White rice (g) & 2124 & 1254 & 420 & 480 & 1674 \\
\hline Wholegrain crackers (g) & 991 & 585 & 196 & 224 & 781 \\
\hline \multicolumn{6}{|l|}{ Meats, poultry, fish, eggs, nuts and seeds } \\
\hline Beef mince $(\mathrm{g})$ & 1514 & 865 & 303 & 433 & 1168 \\
\hline Lamb loin chops (g) & 1516 & 866 & 303 & 433 & 1169 \\
\hline Beef rump steak (g) & 1518 & 867 & 304 & 434 & 1171 \\
\hline Tuna, canned in springwater (g) & 2675 & 1374 & 467 & 934 & 1841 \\
\hline Chicken breast (g) & 2137 & 1098 & 373 & 746 & 1471 \\
\hline Eggs (g) & 3208 & 1648 & 560 & 1120 & 2208 \\
\hline Peanuts, unsalted (g) & 960 & 360 & 420 & 420 & 780 \\
\hline \multicolumn{6}{|l|}{ Milk, yoghurt, cheese and alternatives } \\
\hline Cheddar cheese, full fat (g) & 1104 & 544 & 160 & 520 & 704 \\
\hline Milk, full fat (ml) & 7651 & 3713 & 1125 & 3375 & 4838 \\
\hline Cheddar cheese, reduced fat (g) & 408 & 198 & 60 & 180 & 258 \\
\hline Milk, reduced fat (ml) & 26867 & 13034 & 4000 & 12167 & 17034 \\
\hline Yoghurt, reduced fat (g) & 10746 & 5213 & 1600 & 4867 & 6813 \\
\hline \multicolumn{6}{|l|}{ Unsaturated oils and spreads } \\
\hline Canola margarine (g) & 538 & 226 & 186 & 186 & 412 \\
\hline Sunflower oil (ml) & 757 & 318 & 261 & 262 & 579 \\
\hline
\end{tabular}

HH Household

${ }^{\mathrm{a}} \mathrm{HH} 1(n=6)$ : male $19-50$ year; female $19-50$ year; female $70+$ yrs; boy 14 years; girl 8 years; boy 4 years

${ }^{\mathrm{b}} \mathrm{HH} 2(n=3)$ : female $19-50$ year; boy 14 years; girl 8 years

${ }^{\mathrm{C}} \mathrm{HH} 3(n=1)$ : male $19-50$ year

${ }^{\mathrm{d}} \mathrm{HH} 4(n=2)$ : male $70+\mathrm{yrs}$; female $70+\mathrm{yrs}$

${ }^{\mathrm{e}} \mathrm{HH} 5(n=4)$ : adult male $19-50$ year old; adult female $19-50$ year old; boy 14 years old; girl 8 years 
Table 5 Price of healthy (recommended) and current (unhealthy) diet baskets for five household structures at two locations in Queensland

Location

Diet Basket Components

Total diet basket

\$mean \pm sd

Fruit and Vegetables

$\$$ mean \pm sd

(\% total basket price)

Core five food group

foods \$mean \pm sd

(\% total Basket price)

Fruit \$mean \pm sd

(\% total basket price)

Vegetables \& legumes/

beans $\$$ mean \pm sd

(\% total basket)

Grains (cereal) foods

$\$$ mean \pm sd

(\% total basket price)

Lean meats and poultry,

fish eggs, tofu, nuts, seeds,

legumes/beans \$mean \pm sd

(\% total basket price)

Milk, yoghurt, cheese and/ or alternatives $\$$ mean $\pm \mathrm{sd}$

(\% total basket price)

Unsaturated Oils \& Spreads \$mean \pm sd (\% total basket price)

All discretionary choices \$mean \pm sd (\% tota

basket price)

Alcoholic drinks \$mean \pm sd (\% total basket price)

Take-away foods \$mean \pm sd (\% total basket price)

Sugar-sweetened

beverages

$\$$ mean \pm sd

(\% total basket price)

\begin{tabular}{|c|c|c|c|c|c|c|c|c|c|}
\hline $\begin{array}{l}\mathrm{HH} 1 \text { Healthy } \\
\text { diet }\end{array}$ & $\begin{array}{l}\mathrm{HH} 1 \text { Current } \\
\text { (unhealthy) diet }\end{array}$ & $\begin{array}{l}\mathrm{HH} 2 \text { Healthy } \\
\text { Diet }\end{array}$ & $\begin{array}{l}\mathrm{HH} 2 \text { Current } \\
\text { (unhealthy) diet }\end{array}$ & $\begin{array}{l}\mathrm{HH} 3 \\
\text { Healthy Diet }\end{array}$ & $\begin{array}{l}\text { HH } 3 \text { Current } \\
\text { (unhealthy) diet }\end{array}$ & $\begin{array}{l}\text { HH } 4 \\
\text { Healthy Diet }\end{array}$ & $\begin{array}{l}\mathrm{HH} 4^{\mathrm{a}} \text { Current } \\
\text { (unhealthy) diet }\end{array}$ & $\begin{array}{l}\text { HH } 5 \text { Healthy } \\
\text { diet }\end{array}$ & $\begin{array}{l}\text { HH } 5 \text { Current } \\
\text { (unhealthy) diet }\end{array}$ \\
\hline $795.47 \pm 25.76$ & $896.04 \pm 5.28$ & $414.50 \pm 13.66$ & $418.71 \pm 1.59$ & $146.43 \pm 4.53$ & $221.83 \pm 1.22$ & $272.88 \pm 8.67$ & $325.99 \pm 2.42$ & $560.93 \pm 18.19$ & $640.20 \pm 2.67$ \\
\hline $\begin{array}{c}239.43 \pm 19.11 \\
(30.10 \%)\end{array}$ & $\begin{array}{c}120.22 \pm 6.89 \\
(13.42 \%)\end{array}$ & $\begin{array}{c}121.61 \pm 9.82 \\
(29.34 \%)\end{array}$ & $\begin{array}{l}61.30 \pm 3.69 \\
(14.64 \%)\end{array}$ & $\begin{array}{l}44.95 \pm 3.38 \\
(30.70 \%)\end{array}$ & $\begin{array}{l}21.62 \pm 1.14 \\
(9.75 \%)\end{array}$ & $\begin{array}{c}80.91 \pm 6.72 \\
(29.65 \%)\end{array}$ & $\begin{array}{c}46.38 \pm 2.54 \\
(14.23 \%)\end{array}$ & $\begin{array}{c}166.56 \pm 13.20 \\
(29.69 \%)\end{array}$ & $\begin{array}{c}82.92 \pm 4.83 \\
(12.95 \%)\end{array}$ \\
\hline $\begin{array}{c}795.47 \pm 25.76 \\
(100.0 \%)\end{array}$ & $\begin{array}{c}386.95 \pm 11.95 \\
(43.19 \%)\end{array}$ & $\begin{array}{c}414.50 \pm 13.66 \\
(100.0 \%)\end{array}$ & $\begin{array}{c}188.20 \pm 5.88 \\
(44.95 \%)\end{array}$ & $\begin{array}{c}146.43 \pm 4.53 \\
(100.0 \%)\end{array}$ & $\begin{array}{l}80.25 \pm 2.55 \\
(36.18 \%)\end{array}$ & $\begin{array}{c}272.88 \pm 8.67 \\
(100.0 \%)\end{array}$ & $\begin{array}{c}141.36 \pm 4.39 \\
(43.36 \%)\end{array}$ & $\begin{array}{c}560.93 \pm 18.19 \\
(100.0 \%)\end{array}$ & $\begin{array}{c}268.06 \pm 8.40 \\
(41.87 \%)\end{array}$ \\
\hline $\begin{array}{c}107.78 \pm 17.70 \\
(13.55 \%)\end{array}$ & $\begin{array}{c}71.65 \pm 6.40 \\
(8.00 \%)\end{array}$ & $\begin{array}{l}55.32 \pm 9.08 \\
(13.35 \%)\end{array}$ & $\begin{array}{c}38.79 \pm 3.48 \\
(9.26 \%)\end{array}$ & $\begin{array}{l}19.08 \pm 3.13 \\
(13.03 \%)\end{array}$ & $\begin{array}{l}11.41 \pm 1.02 \\
(5.14 \%)\end{array}$ & $\begin{array}{c}38.15 \pm 6.26 \\
(13.98 \%)\end{array}$ & $\begin{array}{c}26.02 \pm 2.31 \\
(7.98 \%)\end{array}$ & $\begin{array}{c}74.39 \pm 12.22 \\
(13.26 \%)\end{array}$ & $\begin{array}{l}50.20 \pm 4.50 \\
\quad(7.84 \%)\end{array}$ \\
\hline $\begin{array}{c}131.65 \pm 3.47 \\
(16.55 \%)\end{array}$ & $\begin{array}{l}48.57 \pm 0.50 \\
\quad(5.42 \%)\end{array}$ & $\begin{array}{c}66.29 \pm 1.76 \\
(15.99 \%)\end{array}$ & $\begin{array}{c}22.51 \pm 0.21 \\
(5.37 \%)\end{array}$ & $\begin{array}{l}25.88 \pm 0.52 \\
(17.67 \%)\end{array}$ & $\begin{array}{l}10.21 \pm 0.13 \\
(4.60 \%)\end{array}$ & $\begin{array}{l}42.76 \pm 1.18 \\
(15.67 \%)\end{array}$ & $\begin{array}{c}20.36 \pm 0.27 \\
(6.25 \%)\end{array}$ & $\begin{array}{c}92.17 \pm 2.28 \\
(16.43 \%)\end{array}$ & $\begin{array}{c}32.72 \pm 0.33 \\
\quad(5.11 \%)\end{array}$ \\
\hline $\begin{array}{c}132.07 \pm 0.24 \\
(16.60 \%)\end{array}$ & $\begin{array}{c}67.29 \pm 1.83 \\
(7.51 \%)\end{array}$ & $\begin{array}{c}76.10 \pm 0.14 \\
(18.36 \%)\end{array}$ & $\begin{array}{c}32.97 \pm 0.85 \\
(7.87 \%)\end{array}$ & $\begin{array}{c}25.67 \pm 0.05 \\
(17.53 \%)\end{array}$ & $\begin{array}{l}13.050 .43 \\
(5.88 \%)\end{array}$ & $\begin{array}{c}32.12 \pm 0.06 \\
(11.77 \%)\end{array}$ & $\begin{array}{c}22.54 \pm 0.65 \\
(6.91 \%)\end{array}$ & $\begin{array}{c}101.78 \pm 0.19 \\
(18.14 \%)\end{array}$ & $\begin{array}{c}46.02 \pm 1.28 \\
(7.19 \%)\end{array}$ \\
\hline $\begin{array}{c}252.02 \pm 8.73 \\
(31.68 \%)\end{array}$ & $\begin{array}{c}143.48 \pm 6.80 \\
(16.01 \%)\end{array}$ & $\begin{array}{c}133.76 \pm 5.00 \\
(32.27 \%)\end{array}$ & $\begin{array}{l}66.53 \pm 3.04 \\
(15.89 \%)\end{array}$ & $\begin{array}{l}48.64 \pm 1.40 \\
(33.21 \%)\end{array}$ & $\begin{array}{l}35.63 \pm 1.78 \\
(16.06 \%)\end{array}$ & $\begin{array}{l}82.72 \pm 2.56 \\
(30.32 \%)\end{array}$ & $\begin{array}{l}55.96 \pm 2.57 \\
(17.16 \%)\end{array}$ & $\begin{array}{c}182.39 \pm 6.38 \\
(32.52 \%)\end{array}$ & $\begin{array}{c}101.77 \pm 4.80 \\
\quad(15.90 \%)\end{array}$ \\
\hline $\begin{array}{c}160.00 \pm 3.33 \\
(20.54 \%)\end{array}$ & $\begin{array}{l}54.39 \pm 0.98 \\
(6.07 \%)\end{array}$ & $\begin{array}{c}79.42 \pm 1.62 \\
(19.16 \%)\end{array}$ & $\begin{array}{c}26.75 \pm 0.49 \\
(6.39 \%)\end{array}$ & $\begin{array}{c}24.20 \pm 0.50 \\
(16.53 \%)\end{array}$ & $\begin{array}{l}9.62 \pm 0.16 \\
(4.34 \%)\end{array}$ & $\begin{array}{l}74.15 \pm 1.51 \\
(27.17 \%)\end{array}$ & $\begin{array}{c}15.648 \pm 0.28 \\
(4.80 \%)\end{array}$ & $\begin{array}{c}103.62 \pm 2.11 \\
(18.47 \%)\end{array}$ & $\begin{array}{c}36.37 \pm 0.65 \\
(5.68 \%)\end{array}$ \\
\hline \multirow[t]{5}{*}{$\begin{array}{l}8.59 \pm 0.28 \\
(1.08 \%)\end{array}$} & $\begin{array}{l}1.57 \pm 0.04 \\
(0.18 \%)\end{array}$ & $\begin{array}{c}3.61 \pm 0.12 \\
(0.87 \%)\end{array}$ & $\begin{array}{l}0.65 \pm 0.02 \\
(0.16 \%)\end{array}$ & $\begin{array}{l}2.97 \pm 0.10 \\
(2.03 \%)\end{array}$ & $\begin{array}{l}0.33 \pm 0.01 \\
(0.15 \%)\end{array}$ & $\begin{array}{l}2.97 \pm 0.10 \\
(1.09 \%)\end{array}$ & $\begin{array}{l}0.85 \pm 0.02 \\
(0.26 \%)\end{array}$ & $\begin{array}{l}6.58 \pm 0.22 \\
(1.17 \%)\end{array}$ & $\begin{array}{l}0.98 \pm 0.03 \\
(0.15 \%)\end{array}$ \\
\hline & $\begin{array}{c}509.08 \pm 17.16 \\
(56.81 \%)\end{array}$ & & $\begin{array}{c}230.51 \pm 7.36 \\
(55.05 \%)\end{array}$ & & $\begin{array}{l}141.58 \pm 3.76 \\
(63.82 \%)\end{array}$ & & $\begin{array}{c}184.63 \pm 6.81 \\
(56.64 \%)\end{array}$ & & $\begin{array}{c}372.13 \pm 11.04 \\
(58.13 \%)\end{array}$ \\
\hline & $\begin{array}{c}117.89 \pm 0.62 \\
(13.16 \%)\end{array}$ & & $\begin{array}{c}40.72 \pm 0.22 \\
(9.73 \%)\end{array}$ & & $\begin{array}{l}49.61 \pm 0.38 \\
(22.36 \%)\end{array}$ & & $\begin{array}{c}66.43 \pm 0.65 \\
(20.38 \%)\end{array}$ & & $\begin{array}{c}90.33 \pm 0.59 \\
(14.11 \%)\end{array}$ \\
\hline & $\begin{array}{c}123.63 \pm 0.00 \\
(13.80 \%)\end{array}$ & & $\begin{array}{l}62.19 \pm 0.00 \\
(14.85 \%)\end{array}$ & & $\begin{array}{l}31.53 \pm 0.00 \\
(14.21 \%)\end{array}$ & & $\begin{array}{c}31.59 \pm 0.00 \\
(9.69 \%)\end{array}$ & & $\begin{array}{c}93.49 \pm 0.00 \\
(14.60 \%)\end{array}$ \\
\hline & $\begin{array}{c}31.52 \pm 0.00 \\
(3.52 \%)\end{array}$ & & $\begin{array}{c}16.67 \pm 0.00 \\
(3.98 \%)\end{array}$ & & $\begin{array}{l}8.99 \pm 0.00 \\
(4.05 \%)\end{array}$ & & $\begin{array}{l}5.11 \pm 0.00 \\
(1.57 \%)\end{array}$ & & $\begin{array}{c}25.65 \pm 0.00 \\
(4.01 \%)\end{array}$ \\
\hline
\end{tabular}


Table 5 Price of healthy (recommended) and current (unhealthy) diet baskets for five household structures at two locations in Queensland (Continued)

\begin{tabular}{|c|c|c|c|c|c|c|c|c|c|c|}
\hline \multirow{2}{*}{$\begin{array}{l}\text { Location } \\
\text { Diet Basket Components }\end{array}$} & \multicolumn{10}{|l|}{ Indooroopilly } \\
\hline & $\begin{array}{l}\text { HH } 1 \text { Healthy } \\
\text { diet }\end{array}$ & $\begin{array}{l}\text { HH } 1 \text { Current } \\
\text { (unhealthy) diet }\end{array}$ & $\begin{array}{l}\text { HH } 2 \text { Healthy } \\
\text { Diet }\end{array}$ & $\begin{array}{l}\text { HH } 2 \text { Current } \\
\text { (unhealthy) diet }\end{array}$ & $\begin{array}{l}\text { HH } 3 \text { Healthy } \\
\text { Diet }\end{array}$ & $\begin{array}{l}\text { HH } 3 \text { Current } \\
\text { (unhealthy) diet }\end{array}$ & $\begin{array}{l}\text { HH } 4 \text { Healthy } \\
\text { Diet }\end{array}$ & $\begin{array}{l}\mathrm{HH} 4^{\mathrm{a}} \text { Current } \\
\text { (unhealthy) diet }\end{array}$ & $\begin{array}{l}\text { HH } 5 \text { Healthy } \\
\text { diet }\end{array}$ & $\begin{array}{l}\text { HH } 5 \text { Current } \\
\text { (unhealthy) diet }\end{array}$ \\
\hline $\begin{array}{l}\text { Total diet basket } \\
\text { \$mean } \pm \text { sd }\end{array}$ & $822.85 \pm 55.61$ & $925.69 \pm 45.53$ & $428.84 \pm 28.77$ & $432.75 \pm 20.16$ & $151.17 \pm 9.89$ & $229.44 \pm 9.84$ & $282.17 \pm 19.43$ & $336.68 \pm 15.67$ & $580.01 \pm 38.66$ & $661.92 \pm 30.00$ \\
\hline $\begin{array}{l}\text { Fruit and Vegetables } \\
\$ \text { mean } \pm \text { sd } \\
\text { (\% total basket price) }\end{array}$ & $\begin{array}{l}254.02 \pm 28.13 \\
(30.87 \%)\end{array}$ & $\begin{array}{l}124.12 \pm 10.51 \\
(13.41 \%)\end{array}$ & $\begin{array}{c}128.99 \pm 14.32 \\
(30.08 \%)\end{array}$ & $\begin{array}{c}63.35 \pm 5.53 \\
(14.64 \%)\end{array}$ & $\begin{array}{l}47.53 \pm 4.98 \\
(31.44 \%)\end{array}$ & $\begin{array}{l}22.33 \pm 1.87 \\
(9.73 \%)\end{array}$ & $\begin{array}{c}86.06 \pm 9.79 \\
(30.50 \%)\end{array}$ & $\begin{array}{c}47.82 \pm 3.94 \\
(14.20 \%)\end{array}$ & $\begin{array}{c}176.53 \pm 19.30 \\
(30.44 \%)\end{array}$ & $\begin{array}{c}85.68 \pm 7.40 \\
(12.94 \%)\end{array}$ \\
\hline $\begin{array}{l}\text { Core five food group } \\
\text { foods } \$ \text { mean } \pm \text { sd } \\
\text { (\% total Basket price) }\end{array}$ & $\begin{array}{l}822.85 \pm 55.61 \\
(100.0 \%)\end{array}$ & $\begin{array}{c}396.77 \pm 24.47 \\
(42.86 \%)\end{array}$ & $\begin{array}{c}428.84 \pm 28.77 \\
(100.0 \%)\end{array}$ & $\begin{array}{c}192.94 \pm 11.75 \\
(44.58 \%)\end{array}$ & $\begin{array}{l}151.17 \pm 9.89 \\
(100.0 \%)\end{array}$ & $\begin{array}{l}82.31 \pm 5.18 \\
(35.88 \%)\end{array}$ & $\begin{array}{l}282.17 \pm 19.43 \\
(100.0 \%)\end{array}$ & $\begin{array}{l}144.98 \pm 8.84 \\
(43.06 \%)\end{array}$ & $\begin{array}{l}580.01 \pm 38.66 \\
\quad(100.0 \%)\end{array}$ & $\begin{array}{l}274.86 \pm 16.94 \\
(41.53 \%)\end{array}$ \\
\hline $\begin{array}{l}\text { Fruit } \$ \text { mean } \pm \text { sd } \\
(\% \text { total basket price) }\end{array}$ & $\begin{array}{c}119.20 \pm 20.39 \\
(14.49 \%)\end{array}$ & $\begin{array}{l}74.91 \pm 8.86 \\
(8.09 \%)\end{array}$ & $\begin{array}{l}61.18 \pm 10.46 \\
(14.27 \%)\end{array}$ & $\begin{array}{c}40.58 \pm 4.86 \\
(9.38 \%)\end{array}$ & $\begin{array}{l}21.10 \pm 3.61 \\
(13.96 \%)\end{array}$ & $\begin{array}{l}11.93 \pm 1.43 \\
(5.20 \%)\end{array}$ & $\begin{array}{c}42.19 \pm 7.22 \\
(14.95 \%)\end{array}$ & $\begin{array}{c}27.17 \pm 3.14 \\
(8.07 \%)\end{array}$ & $\begin{array}{c}82.28 \pm 14.07 \\
(14.19 \%)\end{array}$ & $\begin{array}{l}52.51 \pm 6.30 \\
(7.93 \%)\end{array}$ \\
\hline $\begin{array}{l}\text { Vegetables \& legumes/ } \\
\text { beans } \$ \text { mean } \pm \text { sd } \\
\text { (\% total basket) }\end{array}$ & $\begin{array}{l}134.82 \pm 9.36 \\
(16.38 \%)\end{array}$ & $\begin{array}{l}49.21 \pm 1.98 \\
\quad(5.32 \%)\end{array}$ & $\begin{array}{l}67.81 \pm 4.66 \\
(15.81 \%)\end{array}$ & $\begin{array}{l}22.77 \pm 0.78 \\
(5.26 \%)\end{array}$ & $\begin{array}{l}26.43 \pm 1.67 \\
(17.49 \%)\end{array}$ & $\begin{array}{l}10.39 \pm 0.52 \\
(4.53 \%)\end{array}$ & $\begin{array}{c}43.87 \pm 3.12 \\
(15.55 \%)\end{array}$ & $\begin{array}{c}20.65 \pm 0.98 \\
(6.13 \%)\end{array}$ & $\begin{array}{l}94.25 \pm 6.34 \\
(16.25 \%)\end{array}$ & $\begin{array}{l}33.16 \pm 1.31 \\
\quad(5.01 \%)\end{array}$ \\
\hline $\begin{array}{l}\text { Grains (cereal) foods } \\
\$ \text { mean } \pm \text { sd } \\
\text { (\% total basket price) }\end{array}$ & $\begin{array}{l}136.17 \pm 6.99 \\
(16.55 \%)\end{array}$ & $\begin{array}{l}68.14 \pm 2.86 \\
(7.36 \%)\end{array}$ & $\begin{array}{c}78.46 \pm 4.01 \\
(18.30 \%)\end{array}$ & $\begin{array}{l}33.39 \pm 1.37 \\
(7.72 \%)\end{array}$ & $\begin{array}{l}26.47 \pm 1.36 \\
(17.51 \%)\end{array}$ & $\begin{array}{l}13.20 \pm 0.61 \\
(5.75 \%)\end{array}$ & $\begin{array}{l}33.13 \pm 1.72 \\
(11.74 \%)\end{array}$ & $\begin{array}{l}22.82 \pm 0.98 \\
(6.78 \%)\end{array}$ & $\begin{array}{l}104.93 \pm 5.37 \\
\quad(18.09 \%)\end{array}$ & $\begin{array}{l}46.59 \pm 1.97 \\
(7.04 \%)\end{array}$ \\
\hline $\begin{array}{l}\text { Lean meats and poultry, } \\
\text { fish eggs, tofu, nuts, seeds, } \\
\text { legumes/beans } \$ \text { mean } \pm \text { sd } \\
\text { (\% total basket price) }\end{array}$ & $\begin{array}{c}257.40 \pm 11.62 \\
(31.28 \%)\end{array}$ & $\begin{array}{c}147.08 \pm 8.27 \\
(15.89 \%)\end{array}$ & $\begin{array}{c}136.76 \pm 6.27 \\
(31.89 \%)\end{array}$ & $\begin{array}{c}68.04 \pm 3.46 \\
(15.72 \%)\end{array}$ & $\begin{array}{l}49.45 \pm 2.15 \\
(32.71 \%)\end{array}$ & $\begin{array}{l}36.59 \pm 2.21 \\
(15.95 \%)\end{array}$ & $\begin{array}{c}84.38 \pm 3.72 \\
(29.90 \%)\end{array}$ & $\begin{array}{l}57.42 \pm 3.08 \\
(17.06 \%)\end{array}$ & $\begin{array}{l}186.21 \pm 8.43 \\
(32.11 \%)\end{array}$ & $\begin{array}{l}104.24 \pm 5.68 \\
\quad(15.75 \%)\end{array}$ \\
\hline $\begin{array}{l}\text { Milk, yoghurt, cheese and/ } \\
\text { or alternatives } \$ \text { mean } \pm \text { sd } \\
\text { (\% total basket price) }\end{array}$ & $\begin{array}{l}166.35 \pm 10.84 \\
(20.22 \%)\end{array}$ & $\begin{array}{l}55.80 \pm 3.61 \\
(6.03 \%)\end{array}$ & $\begin{array}{l}80.88 \pm 5.28 \\
(18.86 \%)\end{array}$ & $\begin{array}{l}27.47 \pm 1.83 \\
(6.35 \%)\end{array}$ & $\begin{array}{l}24.64 \pm 1.60 \\
(16.30 \%)\end{array}$ & $\begin{array}{l}9.86 \pm 0.60 \\
(4.30 \%)\end{array}$ & $\begin{array}{l}75.52 \pm 4.95 \\
(26.76 \%)\end{array}$ & $\begin{array}{c}16.03 \pm 1.01 \\
(4.76 \%)\end{array}$ & $\begin{array}{l}105.52 \pm 6.88 \\
(18.19 \%)\end{array}$ & $\begin{array}{l}37.33 \pm 2.43 \\
(5.64 \%)\end{array}$ \\
\hline $\begin{array}{l}\text { Unsaturated Oils \& Spreads } \\
\text { \$mean } \pm \text { sd (\% total basket } \\
\text { price) }\end{array}$ & $\begin{array}{l}8.91 \pm 0.83 \\
(1.08 \%)\end{array}$ & $\begin{array}{l}1.64 \pm 0.16 \\
(0.18 \%)\end{array}$ & $\begin{array}{l}3.74 \pm 0.35 \\
(0.87 \%)\end{array}$ & $\begin{array}{l}0.68 \pm 0.07 \\
(0.16 \%)\end{array}$ & $\begin{array}{l}3.08 \pm 0.29 \\
(2.03 \%)\end{array}$ & $\begin{array}{l}0.34 \pm 0.03 \\
(0.15 \%)\end{array}$ & $\begin{array}{l}3.08 \pm 0.29 \\
(1.09 \%)\end{array}$ & $\begin{array}{l}0.89 \pm 0.09 \\
(0.26 \%)\end{array}$ & $\begin{array}{l}6.82 \pm 0.63 \\
(1.18 \%)\end{array}$ & $\begin{array}{l}1.02 \pm 0.10 \\
(0.15 \%)\end{array}$ \\
\hline $\begin{array}{l}\text { All discretionary choices } \\
\$ \text { mean } \pm \text { sd (\% total } \\
\text { basket price) }\end{array}$ & & $\begin{array}{l}528.93 \pm 21.85 \\
\quad(57.14 \%)\end{array}$ & & $\begin{array}{l}239.81 \pm 9.69 \\
\quad(55.42 \%)\end{array}$ & & $\begin{array}{l}147.12 \pm 5.23 \\
(64.12 \%)\end{array}$ & & $\begin{array}{l}191.70 \pm 8.05 \\
(56.94 \%)\end{array}$ & & $\begin{array}{c}387.05 \pm 14.92 \\
(58.47 \%)\end{array}$ \\
\hline $\begin{array}{l}\text { Alcoholic drinks } \$ \text { mean } \pm \\
\text { sd ( } \% \text { total basket price) }\end{array}$ & & $\begin{array}{l}116.92 \pm 0.12 \\
(12.63 \%)\end{array}$ & & $\begin{array}{l}40.41 \pm 0.03 \\
(9.34 \%)\end{array}$ & & $\begin{array}{l}49.23 \pm 0.21 \\
(21.46 \%)\end{array}$ & & $\begin{array}{l}65.76 \pm 0.63 \\
(19.53 \%)\end{array}$ & & $\begin{array}{l}89.64 \pm 0.24 \\
(13.54 \%)\end{array}$ \\
\hline $\begin{array}{l}\text { Take-away foods } \$ \text { mean } \pm \\
\text { sd (\% total basket price) }\end{array}$ & & $\begin{array}{l}135.35 \pm 0.00 \\
\quad(14.62 \%)\end{array}$ & & $\begin{array}{l}68.14 \pm 0.00 \\
(15.74 \%)\end{array}$ & & $\begin{array}{l}34.42 \pm 0.00 \\
(15.00 \%)\end{array}$ & & $\begin{array}{l}35.10 \pm 0.00 \\
(10.42 \%)\end{array}$ & & $\begin{array}{l}102.28 \pm 0.00 \\
\quad(15.45 \%)\end{array}$ \\
\hline $\begin{array}{l}\text { Sugar-sweetened } \\
\text { beverages } \\
\$ \text { mean } \pm \text { sd } \\
\text { ( } \% \text { total basket price) }\end{array}$ & & $\begin{array}{l}32.01 \pm 0.85 \\
(3.46 \%)\end{array}$ & & $\begin{array}{l}16.93 \pm 0.45 \\
(3.91 \%)\end{array}$ & & $\begin{array}{l}9.13 \pm 0.24 \\
(3.98 \%)\end{array}$ & & $\begin{array}{l}5.19 \pm 0.14 \\
(1.54 \%)\end{array}$ & & $\begin{array}{l}26.04 \pm 0.69 \\
(3.93 \%)\end{array}$ \\
\hline
\end{tabular}


11-18\% required); lean meats, poultry, fish, eggs and plant-based alternatives (16-17\% currently compared to 30-33\% required); milk, cheese, yoghurt (4-6 \% currently compared to $17-27 \%$ required); and unsaturated oils and spreads $(<0.3 \%$ currently compared to $1-2 \%$ required).

Worryingly, the majority of the food dollar is being spent on discretionary choices at 53-64\% in both high and low socio-economic locations (Table 5). Within this category, $10-22 \%$ of the total food dollar is spent on alcoholic drinks; this proportion is lowest in households containing children. If alcoholic drinks are excluded from the current diet, the cost of the healthy (recommended) diets is between 2-8\% more expensive than the current diet for all households except that of the single male (HH3). At $10-16 \%$ of the current food dollar, spending is also relatively high on take-away foods (such as hamburgers and pizzas) and on sugar-sweetened beverages (1.6-4 \%); spending for both these items is highest in families with children.

\section{Testing the impact of policy change on diet prices}

In one example of policy change tested, proposal to expand the base of the GST to include fresh healthy food in Australia, the cost of the healthy (recommended) diet basket would significantly increase by $9.9 \%$ but the cost of the current (unhealthy) diet basket would increase by only $4.5 \%$ to $5.5 \%(\mathrm{Z}=-4.78, P<0.01)$ (Table 6$)$. In real terms, in order to purchase a healthy diet, on average the various households would need to find additional funds of: $\$ 80.15$ for $\mathrm{HH} 1 ; \$ 41.86$ for $\mathrm{HH} 2 ; \$ 14.52$ for HH3; $\$ 27.40$ for HH4; and $\$ 56.39$ for HH5, whereas the additional cost of current (unhealthy) diets would increase by less than $45 \%$ of these amounts. Under this scenario, healthy diets would become relatively more expensive than current (unhealthy) diets, and the discretionary items in the current (unhealthy diet) would become relatively more affordable also.

If the GST base remained the same but the rate of GST was increased to $15 \%$ or $20 \%$ respectively, the price of the healthy diet basket would be similar to present, but the prices of the current (unhealthy) diet basket would increase by around $2 \%$ and $4-5 \%$ respectively, with the discretionary items in the current basket increasing by about twice these rates. Under the latter scenario, healthy diets would become relatively less expensive than current (unhealthy) diets.

\section{Affordability}

The median family income per fortnight in 2011 was $\$ 4,342$ in the high SES area and $\$ 2740$ per fortnight in the low SES area [28]. In the high SES area, the cost of the healthy and current (unhealthy) diet baskets for the average family household per fortnight is \$610.56 (14\% of median income) and $\$ 673.45$ (15.5\% of median income) respectively. In the low SES area, the cost of the healthy and current (unhealthy) diet baskets for the average family household per fortnight is \$590 [28] (21.5\% of median income) and $\$ 651.65$ (23.7\% of median income) respectively.

The affordability of the diet baskets in low income households is nearly half that of families of median income (Table 6). Affordability of the healthy diet basket as a proportion of household income ranges from around $20 \%$ for the low income household of two pensioners (HH4) to around $30 \%$ for the low income household of six (HH1).

However, for these low income families, under the mooted changes to expand the base of the GST to include basic healthy food in Australia, the affordability of the healthy (recommended) diet would decrease by around $4 \%$, but the affordability of their current (unhealthy) diet would decrease by only around $2 \%$ (Table 6). However, if the GST exemption on basic healthy foods was retained, but the rate on other foods and goods and services was increased by $15 \%$ or $20 \%$,a healthy diet would be $7 \%$ and $9 \%$ respectively more relatively affordable than an unhealthy diet (Table 6); but the pressure on the food budget would likely be increased due to the increased cost of other essential items [29].

\section{Discussion}

Price and price differential of healthy (recommended) and current (unhealthy) diets

This study aimed to pilot the development and testing of draft tools, survey protocols, data collection and analysis systems to investigate the price, relative price and affordability of healthy (recommended) and current (unhealthy) diets in Australia, as well as test the utility of the approach to assess impacts of potential policy changes on diet prices, in this case the proposed broadening and/or raising of the GST base in Australia.

Findings suggest that healthy diets consistent with national dietary recommendations are less expensive than current (unhealthy) diets consumed by the Australian population; however lower income households are still required to spend a high proportion of their disposable income accessing healthy foods. As there is a common perception that healthy foods are more expensive than unhealthy foods $[7,31,32]$ these results may be considered surprising. A systematic review that accounted for key sources of heterogeneity, found little difference between the prices of healthier versus less healthy dietary patterns, although the former tended to be slightly more expensive [31]. The effect of the cost of alcoholic drinks and pre-prepared 'convenience' foods do not appear to have been considered in that review, which may help 
Table 6 Affordability of healthy (recommended) and current (unhealthy) diet baskets for five household structures for present pricing and projected pricing after potential changes to the Australian taxation system

\begin{tabular}{|c|c|c|c|c|c|c|c|c|c|c|}
\hline \multirow[b]{2}{*}{ Household } & \multicolumn{10}{|l|}{ Logan } \\
\hline & \multicolumn{2}{|l|}{$\mathrm{HH} 1$} & \multicolumn{2}{|l|}{$\mathrm{HH} 2$} & \multicolumn{2}{|l|}{$\mathrm{HH} 3$} & \multicolumn{2}{|l|}{$\mathrm{HH} 4^{\mathrm{a}}$} & \multicolumn{2}{|l|}{$\mathrm{HH} 5$} \\
\hline $\mathrm{HH}$ income $^{\mathrm{b}}(\$)$ & 2679.38 & & 1545.63 & & 611.76 & & 1389.40 & & 2137.32 & \\
\hline Diet & Healthy & $\begin{array}{l}\text { Current } \\
\text { unhealthy }\end{array}$ & Healthy & $\begin{array}{l}\text { Current } \\
\text { unhealthy }\end{array}$ & Healthy & $\begin{array}{l}\text { Current } \\
\text { unhealthy }\end{array}$ & Healthy & $\begin{array}{l}\text { Current } \\
\text { unhealthy }\end{array}$ & Healthy & $\begin{array}{l}\text { Current } \\
\text { unhealthy }\end{array}$ \\
\hline \multicolumn{11}{|c|}{ Current Taxation system (Basic healthy foods exempt from GST) } \\
\hline Cost of diet/fortnight (\$) & 795.47 & 896.04 & 414.50 & 418.71 & 146.43 & 221.83 & 272.88 & 325.99 & 560.93 & 640.20 \\
\hline Affordability of diet (\%) & 29.69 & 33.44 & 26.82 & 27.09 & 23.94 & 36.26 & 19.64 & 23.46 & 26.24 & 29.95 \\
\hline \multicolumn{11}{|l|}{ GST base expanded to include basic healthy foods (10 \%) } \\
\hline Cost of diet/fortnight (\$) & 874.17 & 942.27 & 455.63 & 440.78 & 160.70 & 231.72 & 299.80 & 343.54 & 616.33 & 672.13 \\
\hline Affordability of diet (\%) & 32.63 & 35.17 & 29.47 & 28.96 & 26.27 & 37.88 & 21.58 & 24.73 & 28.84 & 31.44 \\
\hline Extra total cost/ fortnight \$ (\%) & $\begin{array}{l}78.70 \\
(9.89)\end{array}$ & $46.23(5.16)$ & $\begin{array}{l}41.13 \\
(9.92)\end{array}$ & $22.07(5.27)$ & $\begin{array}{l}14.27 \\
(9.75)\end{array}$ & $9.89(4.46)$ & $\begin{array}{l}26.92 \\
(9.87)\end{array}$ & $17.55(5.38)$ & $\begin{array}{l}55.40 \\
(9.88)\end{array}$ & $31.93(4.99)$ \\
\hline Extra cost of core foods /fortnight $\$(\%)$ & $\begin{array}{l}78.70 \\
(9.89)\end{array}$ & $38.70(10.00)$ & $\begin{array}{l}41.13 \\
(9.92)\end{array}$ & $18.82(10.00)$ & $\begin{array}{l}14.27 \\
(9.75)\end{array}$ & $8.02(10.00)$ & $\begin{array}{l}26.92 \\
(9.87)\end{array}$ & $14.14(10.00)$ & $\begin{array}{l}55.40 \\
(9.88)\end{array}$ & $26.81(10.00)$ \\
\hline Extra cost of discretionary foods /fortnight \$ (\%) & 0 & $7.54(1.48)$ & 0 & $3.25(1.41)$ & 0 & $1.87(1.32)$ & 0 & $3.40(1.84)$ & 0 & $5.13(1.38)$ \\
\hline \multicolumn{11}{|c|}{ Basic healthy foods exempt from GST but GST rate increased to $15 \%^{c}$} \\
\hline Cost of diet/fortnight (\$) & 795.85 & 915.11 & 414.64 & 427.36 & 146.60 & 227.31 & 273.05 & 332.69 & 561.24 & 654.33 \\
\hline Affordability of diet (\%) & 29.70 & 34.15 & 26.81 & 27.63 & 23.96 & 37.16 & 19.65 & 23.94 & 26.25 & 30.61 \\
\hline $\begin{array}{l}\text { Extra total cost increase/fortnight if GST increased to } \\
15 \% \$(\%)\end{array}$ & $\begin{array}{l}0.38 \\
(0.05 \%)\end{array}$ & $19.07(2.13 \%)$ & $\begin{array}{l}0.14 \\
(0.03 \%)\end{array}$ & $8.65(2.07 \%)$ & $\begin{array}{l}0.17 \\
(0.12 \%)\end{array}$ & $5.48(2.47 \%)$ & $\begin{array}{l}0.17 \\
(0.06 \%)\end{array}$ & $6.70(2.06 \%)$ & $\begin{array}{l}0.31 \\
(0.06 \%)\end{array}$ & $14.13(2.21 \%)$ \\
\hline \multicolumn{11}{|c|}{ Basic healthy foods exempt from GST but GST rate increased to $20 \%^{\mathrm{c}}$} \\
\hline Cost of diet/fortnight (\$) & 796.24 & 934.82 & 414.78 & 436.36 & 146.76 & 232.90 & 273.21 & 339.54 & 561.55 & 668.91 \\
\hline Affordability of diet (\%) & 29.72 & 34.89 & 26.84 & 28.23 & 23.99 & 38.07 & $19 . .66$ & 24.43 & 26.27 & 31.30 \\
\hline $\begin{array}{l}\text { Extra total cost increase/fortnight if GST increased to } \\
20 \% \$(\%)\end{array}$ & $\begin{array}{l}0.77 \\
(0.10 \%)\end{array}$ & $38.78(4.33 \%)$ & $\begin{array}{l}0.28 \\
(0.07 \%)\end{array}$ & $17.65(4.22 \%)$ & $\begin{array}{l}0.33 \\
(0.23 \%)\end{array}$ & $11.07(4.99 \%)$ & $\begin{array}{l}0.33 \\
(0.12 \%)\end{array}$ & $13.55(4.16 \%)$ & $\begin{array}{l}0.62 \\
(0.11 \%)\end{array}$ & $28.71(4.48 \%)$ \\
\hline
\end{tabular}


Table 6 Affordability of healthy (recommended) and current (unhealthy) diet baskets for five household structures for present pricing and projected pricing after potential changes to the Australian taxation system (Continued)

\begin{tabular}{|c|c|c|c|c|c|c|c|c|c|c|}
\hline \multirow[b]{2}{*}{ Household } & \multicolumn{10}{|c|}{ Indooroopilly } \\
\hline & \multicolumn{2}{|c|}{$\mathrm{HH} 1$} & \multicolumn{2}{|l|}{$\mathrm{HH} 2$} & \multicolumn{2}{|l|}{$\mathrm{HH} 3$} & \multicolumn{2}{|l|}{$\mathrm{HH} 4^{\mathrm{a}}$} & \multicolumn{2}{|l|}{$\mathrm{HH} 5$} \\
\hline $\mathrm{HH}$ income ${ }^{b}(\$)$ & 2679.38 & & 1545.63 & & 611.76 & & 1389.40 & & 2137.32 & \\
\hline Diet & Healthy & $\begin{array}{l}\text { Current } \\
\text { unhealthy }\end{array}$ & Healthy & $\begin{array}{l}\text { Current } \\
\text { unhealthy }\end{array}$ & Healthy & $\begin{array}{l}\text { Current } \\
\text { unhealthy }\end{array}$ & Healthy & $\begin{array}{l}\text { Current } \\
\text { unhealthy }\end{array}$ & Healthy & $\begin{array}{l}\text { Current } \\
\text { unhealthy }\end{array}$ \\
\hline \multicolumn{11}{|c|}{ Current Taxation system (Basic healthy foods exempt from GST) } \\
\hline Cost of diet/fortnight (\$) & 822.85 & 925.69 & 428.84 & 432.75 & 151.17 & 229.44 & 282.17 & 336.68 & 580.01 & 661.92 \\
\hline Affordability of diet (\%) & 30.71 & 34.55 & 27.75 & 28.00 & 24.71 & 37.50 & 20.31 & 24.23 & 27.13 & 30.97 \\
\hline \multicolumn{11}{|l|}{ GST base expanded to include basic healthy foods (10 \%) } \\
\hline Cost of diet/fortnight (\$) & 904.36 & 974.29 & 471.43 & 455.84 & 165.95 & 239.95 & 310.05 & 355.20 & 637.38 & 695.49 \\
\hline Affordability of diet (\%) & 33.75 & 36.36 & 30.50 & 29.49 & 27.13 & 39.22 & 22.31 & 25.56 & 29.82 & 32.54 \\
\hline Extra total cost/ fortnight \$ (\%) & $\begin{array}{l}81.51 \\
(9.90)\end{array}$ & $48.60(5.25)$ & $\begin{array}{l}42.59 \\
(9.93)\end{array}$ & $23.09(5.34)$ & $\begin{array}{l}14.78 \\
(9.78)\end{array}$ & $10.51(4.58)$ & $\begin{array}{l}27.88 \\
(9.88)\end{array}$ & $18.52(5.50)$ & $\begin{array}{l}57.37 \\
(9.89)\end{array}$ & $33.57(5.07)$ \\
\hline Extra cost of core foods /fortnight $\$(\%)$ & $\begin{array}{l}81.51 \\
(9.90)\end{array}$ & $39.67(10.00)$ & $\begin{array}{l}42.59 \\
(9.93)\end{array}$ & $19.29(10.00)$ & $\begin{array}{l}14.78 \\
(9.78)\end{array}$ & $8.23(10.00)$ & $\begin{array}{l}27.88 \\
(9.88)\end{array}$ & $14.50(10.00)$ & $\begin{array}{l}57.37 \\
(9.89)\end{array}$ & $27.49(10.00)$ \\
\hline Extra cost of discretionary foods /fortnight $\$(\%)$ & 0 & $8.92(1.69)$ & 0 & $3.80(1.58)$ & 0 & $2.28(1.55)$ & 0 & $4.02(2.10)$ & 0 & $6.09(1.57)$ \\
\hline \multicolumn{11}{|c|}{ Basic healthy foods exempt from GST but GST rate increased to $15 \%^{\mathrm{c}}$} \\
\hline Cost of diet/fortnight (\$) & 823.20 & 945.02 & 428.97 & 441.56 & 151.32 & 234.99 & 282.33 & 343.42 & 580.29 & 676.28 \\
\hline Affordability of diet (\%) & 30.72 & 35.27 & 27.74 & 28.56 & 24.74 & 38.41 & 20.32 & 24.72 & 27.15 & 31.64 \\
\hline $\begin{array}{l}\text { Extra total cost increase/fortnight if GST increased to } \\
15 \% \$(\%)\end{array}$ & $\begin{array}{l}0.35 \\
(0.04 \%)\end{array}$ & $19.33(2.09 \%)$ & $\begin{array}{l}0.13 \\
(0.03 \%)\end{array}$ & $8.81(2.04 \%)$ & $\begin{array}{l}0.15 \\
(0.10 \%)\end{array}$ & $5.55(2.42 \%)$ & $\begin{array}{l}0.16 \\
(0.06 \%)\end{array}$ & $6.74(2.00 \%)$ & $\begin{array}{l}0.28 \\
(0.05 \%)\end{array}$ & $14.36(2.17 \%)$ \\
\hline \multicolumn{11}{|c|}{ Basic healthy foods exempt from GST but GST rate increased to $20 \%^{c}$} \\
\hline Cost of diet/fortnight (\$) & 823.56 & 965.01 & 429.10 & 450.74 & 151.48 & 240.64 & 282.48 & 350.31 & 580.58 & 691.10 \\
\hline Affordability of diet (\%) & 30.74 & 36.02 & 27.76 & 29.16 & 24.76 & 39.34 & 20.33 & $25 . .21$ & 27.16 & 32.33 \\
\hline $\begin{array}{l}\text { Extra total cost increase/fortnight if GST increased to } \\
20 \% \$(\%)\end{array}$ & $\begin{array}{l}0.71 \\
(0.09 \%)\end{array}$ & $39.32(4.25 \%)$ & $\begin{array}{l}0.26 \\
(0.06 \%)\end{array}$ & $17.99(4.16 \%)$ & $\begin{array}{l}0.31 \\
(0.21 \%)\end{array}$ & $11.20(4.88 \%)$ & $\begin{array}{l}0.31 \\
(0.11 \%)\end{array}$ & $13.63(4.05 \%)$ & $\begin{array}{l}0.57 \\
(0.10 \%)\end{array}$ & $29.18(4.41 \%)$ \\
\hline
\end{tabular}

a Results for current (unhealthy) diet in HH4 should be applied with caution

${ }^{b}$ Calculated low household income per fortnight. Median income per fortnight is $\$ 2,740$ in Logan and $\$ 4,342$ in Indooroopilly

c All increased costs under this scenario pertain to discretionary food 
explain why our findings differ from these results as the current (unhealthy) Australian diet includes a high proportion of these items. Amongst other components of the diet, depending on the unit reported (that is, per energy, weight or serving), the individual price of some healthy foods, particularly meats and dairy foods and vegetables, are relatively expensive compared with energy dense discretionary foods and are also more expensive than core (healthy) cereal-based foods [31, 33].

The high proportion of the food dollar currently being spent on discretionary choices in Australia is of particular concern. For example, a family of two adults and two children (HH5) spends over $58 \%$ of their food dollar on discretionary choices, which provide around $38 \%$ of their total energy intake. A recent report on the Australian Dietary Guidelines food price indexes released by the Australian Government [34] found that $58.2 \%$ of household's food budget in 2014 was spent on discretionary foods and drinks, confirming our findings. Within the discretionary category, we found households are spending around $14 \%$ on alcoholic drinks, around $15 \%$ on take-away foods and around $4 \%$ on sugarsweetened beverages. This suggests that, while food price is important, other factors such as convenience and/or desirability and 'taste', and the determinants of these factors, such as the ubiquitous availability, advertising and marketing of discretionary choices, poor food literacy and cooking skills and busy lifestyles may be more important influences on food choice in Australia [4].

Higher food prices were observed in the higher SES area compared to the lower SES area consistent with previous studies [35].

No attempt was made to control the price of the healthy (recommended) diet baskets and the current (unhealthy) diet baskets for energy, as the diets are constructed on recommended energy levels and actual reported levels of energy respectively, and the energy content of each is a determinant variable that directly affects diet-related health outcomes [4, 7]. Further, as the key exposure variable affecting the life time risk of dietrelated disease is the total diet and dietary patterns, these findings illustrate that studies such as this pilot that compare the cost of actual diets with recommended diets are more pertinent to the health policy debate than the more common, but limited, studies into the relative price of selected 'healthy' and 'unhealthy' foods or single 'optimised' diets [7, 18, 19, 31].

\section{Affordability of healthy (recommended) and current (unhealthy) diets}

Findings suggest that a healthy diet consistent with Australian Dietary Guidelines [4] is presently affordable for families on a median income costing approximately
$18 \%$ of disposable income, but is much less affordable for low income families, costing around $28 \%$ of their household disposable income (Table 6). In comparison, current (unhealthy) diets cost around $20 \%$ of the income of a family of median income and $32 \%$ of the income of low income families (Table 6).

These results are consistent with household expenditure survey data [30] which showed that $16.5 \%$ of average equivalised disposable income was spent on food and drink in all Queensland households, but at 21-41\% of disposable income of low income households, are higher than the equivalised proportion (19.5\%) reported for these households in another study [29]. Disparities are likely to be due to different methodologies, but the amounts spent on food and drink per week in the official reports $[29,30]$ (for example $\$ 79$ per week for an unemployed single person) are very low suggesting very restricted diets, and do not concord with prices of the mean quantities of food and drink reported in the AHS [3].

Our findings are similar to those community studies of the affordability of selected 'healthier' diets (i.e., not necessarily consistent with dietary recommendations) which cost between $28 \%$ and $40 \%$ of the disposable income of lowest income families, compared with $20 \%$ for families on the average income [7] and up to $48 \%$ of income if environmental sustainability of the diet is also considered [5]. In Aboriginal and Torres Strait Islander communities in Australia, depending on location, it has been estimated that at least $34 \%-80 \%$ of the family income is needed to purchase healthy diets $[9,36]$. At least $3.7 \%$ of Australian households report having run out of food in the previous 12 months and not being able to afford to buy more $[3,6]$. This proportion is higher among some groups, affecting more than one in in five Aboriginal and Torres Strait Islanders (22\%) [37], around $11 \%$ of those unemployed and $16 \%$ of rental households [6].

Internationally there is no accepted benchmark for affordability of a healthy diet [7]. One of the proposed equity targets to close the gap on Indigenous disadvantage in Australia was that, by $2018,90 \%$ of Indigenous families could access a healthy food basket for under $25 \%$ of their disposable income [38]. A figure of $25 \%$ seems reasonable as a working estimate given the competing priorities for other essential items in the household budget, such as housing, transport, health services, clothing and utilities [29].

The fact that current (unhealthy) diets including alcoholic drinks cost more than healthy diets in Australia, may mask the fact that, at $28 \%$ of income, healthy diets are already unaffordable for low income families in Australia, and reinforces the notion that, if we are not to worsen diets and increase the prevalence of diet-related chronic disease in Australia, price-elasticities must be 
considered very carefully before food prices are changed in Australia, as proposed under potential changes to the Australian Tax System [20]. Quantitative modelling of the impacts of such strategies requires an understanding of own-price elasticities (how consumption changes with an item's own price) and cross-price elasticities (how consumption of an item changes with changes in the price of another good) in different groups [39, 40]. An additional consideration for food price elasticities is whether the cross-price elasticities are within the same or different food groups [18]. Plausible food price elasticities have been estimated for use in Australia and New Zealand [40] and globally [41] and could be used to model the consumption and consequent health impacts of the changing prices estimated under various policy scenarios in future studies.

The poor quality of the current Australian diet is consistent with high risk of diet-related disease, especially those mediated by obesity, including type 2 diabetes, cardiovascular disease and some cancers [4] and represents a significant threat to Australia's health, economic and broader social systems $[1,4]$. Therefore it is critical that no additional barriers, such as increased cost of healthy foods, are put in the way of Australians choosing healthier foods and diets.

The findings suggest that more needs to be done to promote healthy diets, including disseminating the fact that they can be less expensive than current dietary patterns. Healthy budgeting programs such as FOODcents may be useful in this regard [42]. Affordability of healthy foods among lower income groups could be improved by promoting cheaper options that are still healthy, such as local specials and seasonal or irregular shaped fresh produce. It is also likely that barriers to healthy choices, such as low food literacy and poor cooking skills, also need to be addressed $[4,43]$. However, there is mounting evidence that current "food environments exploit people's biological, psychological, social and economic vulnerabilities, making it easier for them to eat unhealthy foods" [44] and that "regulatory actions from governments and increased efforts from industry and civil society will be necessary to break these vicious cycles" [44].

Testing impact of policy changes on diet prices: the effect of potential changes to the Australian taxation system

Under potential changes to the Australian Tax System [20], if the GST base was extended to encompass basic healthy foods, the cost of healthy (recommended) diets would increase $(9.9 \%)$ by more than double the rate of current (unhealthy) diets (4.5\%), decreasing the relative affordability of healthy foods. In real terms, a healthy diet would cost a family of two adults and two children an additional $\$ 56.39$ per week. At $30 \%$ of disposable household income, this is likely to be unaffordable for low income families. Under this scenario, it would be less likely that the population would move towards healthier eating patterns, as the healthy foods consumed within the current diet would also increase significantly in price compared with discretionary choices; hence it would be likely that an even greater proportion of the food dollar would be spent on discretionary choices ('junk food').

If the GST is not extended to basic healthy foods but is applied at higher rates (15\% and $20 \%)$ to those foods and drinks currently incurring GST, a healthy diet would cost no more than presently, but the cost of the current (unhealthy) diet would increase by more than $\$ 14.00$ (2.2\%) and around \$29.00 (4.5\%) per fortnight respectively for a family of two adults and two children. As the relative affordability of a healthy diet compared with the current (unhealthy) diet would improve under these scenarios, these policy actions effectively work as a tax on unhealthy food [19]. However the cost of non-food items that currently attract GST at $10 \%$ would also increase, potentially leaving less money to be spent on food, highlighting the regressive nature of GST which disproportionally affects those on lower incomes [19]. Consideration of available price elasticities in Australia [40] and recent 'real world' data showing that a $20 \%$ price reduction in fruit and vegetables increased household purchases by $35 \%$ for fruit and $15 \%$ for vegetables [45] suggests that the higher rate of GST would be most effective in helping drive dietary improvements. However, as mentioned previously, careful consideration of factors other than price that influence dietary choices would also be required. In addition, a healthy diet would still cost up to $28 \%$ of the disposable income of low income families and would be unaffordable for some groups. The regressive nature of taxation on unhealthy foods is potentially problematic but can be alleviated by targeted subsidies to vulnerable groups to help alleviate pressure on the household budget [7, 19]. Differential tax rates, such as $30 \%$ GST on sugar-sweetened beverages, may also have merit [19].

\section{Limitations}

There are several methodological limitations in this study, including that the cost of the current (unhealthy) diet may not be the same as actual expenditure on food, given that it is based on national mean dietary intakes. Other assumptions include that food is shared equitably throughout the households, that there is no home food production and no wastage. Nutritionally similar products were aggregated to minimize the number of items included in the food pricing basket tools but products were not necessarily homogenous in terms of price; similar food items were included in each basket to try to minimize any unintended effects. No adjustments were 
made for costs such as transport, time, cooking equipment and utilities; as these apply to both the current and healthy (recommended) diets, assessment of the price differential between the two can control for some of these hidden costs to some extent, but the effect of these would increase actual diet costs and decrease affordability of the diets. No adjustments were made to account for the marked underreporting in the AHS 2011-12 [3, 37], reported dietary variability amongst different groups other than age/gender stratification, the greater proportion of pre-prepared 'convenience' items containing meats and/or vegetables the current (unhealthy) diet tool, or, given the high rates of overweight/obesity in Australia, that the Foundation diets were prescribed for the shortest and least active in each age group [17]. The study design presumes that price and healthfulness are key drivers of dietary choice in families, but as the results suggest, other needs such as taste and convenience, may now play a greater role in food choice.

Arbitrary decision points occur around sampling frameworks, data collection protocols, analysis and presentation of results, data sources and definitions of family and household income and composition. Such methodological limitations are common to other food price studies, and in order for final methods to be replicable, detailed publication of all assumptions and protocols, and the underlying rationale of the final methods is required.

\section{Next steps}

Our next step is to apply insights from this pilot study to finalize the diet basket pricing tools. We are reconstructing the composition of the current (unhealthy) baskets using detailed unit record data (CURFS) from the AHS 2011-12 [3], analyzing these with the AUSNUT 2011-13 food composition data base and documenting key decision points in the survey protocols to produce a detailed methods paper for the final tools.

Once the tools are finalized, a workshop with all stakeholders will be held to build on previous understandings and seek agreement for the use of these methods as a standardized approach throughout Australia. Pending funding, additional data will be collected in New South Wales, the Australian Capital Territory, Queensland and, ideally, nationally as part of the first INFORMAS assessment of Australian food environments.

Resultant food price and affordability data will be used to feed into broader computer modelling systems, for example, to determine more robust estimates of projected health impacts and costs under different fiscal policy scenarios.

\section{Conclusion}

This pilot study confirms that, to inform fiscal and health policy actions and ensure equitable affordability of healthy diets, standardized food price assessment, monitoring and surveillance efforts should seek to determine and compare the costs of current (unhealthy) diets as well as healthy (recommended) diets. Our findings suggest that the approach suggested in this paper is a valid means of achieving this.

The results show that Australians are spending more on current (unhealthy) diets than would be required to purchase healthy diets consistent with the recommendations of the Australian Dietary Guidelines. Using the methods to test the price impacts of policy options, showed that proposed extension to the GST base to include basic healthy food would markedly increase the cost of a healthy diet relative to current (unhealthy) diets, limiting the affordability of healthy dietary patterns, particularly for lower income households who are already most vulnerable to poor dietrelated health.

Findings also suggest that more needs to be done to tackle assumptions that healthy diets are more expensive than unhealthy diets. More research is required to better understand the relative importance of factors affecting food choice in Australia.

The study has demonstrated that meaningful food pricing tools based on recent Australian dietary data (current unhealthy diet) and recommendations (healthy diet) can be drafted and applied readily. The draft tools appeared fit for purpose and performed well at face value at household level, but some improvements can and will be made. The tools have the potential to be standardized nationally and inform similar research in other countries.

\section{Ethics and consent to participate}

The QUT University Human Research Ethics Committee assessed this study as meeting the conditions for exemption from Human Research Ethics Committee review and approval in accordance with section 5.1.22 of the National Statement on Ethical Conduct in Human Research (2007); the exemption number is 1500000161 . All data were obtained from publically available sources and did not involve human participants, so consent to participate is not applicable.

\section{Consent to publish statement \\ Not applicable.}

\section{Availability of data and materials \\ Data sets have been included as Additional file 1.}

\section{Additional file}

Additional file 1: Detailed calculation of diet basket contents and price. (XLSX $485 \mathrm{~kb})$

Abbreviations

ABS: Australian Bureau of Statistics; AHS: Australian Health Survey; CURFS: Confidentialised Unit Record Files; GST: Goods and Services Tax; IGA: Independent Grocers Australia; INFORMAS: International Network for 
Food and Obesity/non-communicable diseases Research Monitoring and Action Support; NCDs: non-communicable diseases; NUTTAB: Nutrient Tables for use in Australia; PAL: physical activity level; QCOSS: Queensland council of social services; SA2: statistical area level 2; SES: socio-economic status.

\section{Competing interests}

The authors have no competing interests to declare.

\section{Authors' contributions}

$\mathrm{AL}$ designed, led and coordinated the project, designed the project methodology, organized and analyzed the data and drafted and finalized the manuscript. SK participated in the design of the project methodology, collected food prices, organized and analyzed the data and reviewed the manuscript. RR performed the statistical analysis and reviewed the manuscript. EG and MD participated in the design of the project methodology, calculated household income and reviewed the manuscript. All authors read and approved the final manuscript.

\section{Authors' information}

AL leads the INFORMAS food prices and affordability module.

\section{Acknowledgements}

This research was supported by The Australian Prevention Partnership Centre through the NHMRC partnership centre grant scheme (Grant ID: GNT9100001) with the Australian Government Department of Health, NSW Ministry of Health, ACT Health, HCF, and the HCF Research Foundation. The authors would like to thank Christina Pollard and Tim Landrigan for their involvement in conceptual discussions and ongoing support.

\section{Author details}

'School of Public Health and Social Work, Queensland University of Technology, Brisbane, QLD, Australia. ${ }^{2}$ School of Exercise and Nutrition Sciences, Queensland University of Technology, Brisbane, QLD, Australia. ${ }^{3}$ Preventive Health Branch, Prevention Division, Department of Health, Brisbane, QLD, Australia.

\section{Received: 23 October 2015 Accepted: 1 April 2016 Published online: 12 April 2016}

\section{References}

1. Institute for Health Metrics and Evaluation. Global Burden of Disease Country Profile Australia. 2013. http://www.healthdata.org/sites/default/files/ files/country_profiles/GBD/ihme_gbd_country_report_australia.pdf. Accessed 14 Jun 2015

2. Swinburn B, Sacks G, Vandevijvere S, Kumanyika S, Lobstein T, Neal B, et al. INFORMAS (International Network for Food and Obesity/non-communicable diseases Research, Monitoring and Action Support): overview and key principles. Obes Rev. 2013;14(S1):1-12.

3. Australian Bureau of Statistics. Australian Health Survey: Nutrition First Results - Foods and Nutrients, 2011-12. Canberra: ABS. 2014. http://www. abs.gov.au/ausstats/abs@.nsf/Lookup/4364.0.55.007main+features22011-12. Accessed 11 Feb 2016

4. National Health and Medical Research Council. Australian Dietary Guidelines providing the scientific evidence for healthier Australian Diets. 2013. https:// www.eatforhealth.gov.au/sites/default/files/files/the_guidelines/n55_australian_ dietary_guidelines.pdf. Accessed 9 Feb 2016

5. Barosh L, Friel S, Engelhardt K, Chan L. The cost of a healthy and sustainable diet-who can afford it? Aust N Z J Public Health. 2014:38(1):7-12.

6. Ward PR, Verity F, Carter P, Tsourtos G, Coveney J, Wong KC. Food stress in Adelaide: The relationship between low income and the affordability of healthy food. J Environ Public Health. 2013;2013(1248):1-10.

7. Lee A, Mhurchu CN, Sacks G, Swinburn B, Snowdon W, Vandevijvere S, et al. Monitoring the price and affordability of foods and diets globally. Obes Rev. 2013;14(S1):82-95.

8. Chapman K, Kelly B, Bauman A, Innes-Hughes C, Allman-Farinelli M. Trends in the cost of a healthy food basket and fruit and vegetable availability in New South Wales, Australia, between 2006 and 2009. Nutr Diet. 2014;71(2):117-26.

9. Department of Health NT. Northern Territory Market Basket Survey 2014 Casuarina: Department of Health; 2014. p. 38.
10. Harrison M, Lee A, Findlay M, Nicholls R, Leonard D, Martin C. The increasing cost of healthy food. Aust N Z J Public Health. 2010;34(2):179-86.

11. Kettings C, Sinclair AJ, Voevodin M. A healthy diet consistent with Australian health recommendations is too expensive for welfare-dependent families. Aust N Z J Public Health. 2009;33(6):566-72.

12. Palermo C, Wilson A. Development of a healthy food basket for Victoria. Aust N Z J Public Health. 2007:31(4):360-3.

13. Pattieson D, Palermo C. Summary of 2010 Healthy Food Basket data across various Local Government Areas in Victoria. 2010. http://www.med. monash.edu.au/scs/nutrition-dietetics/documents/2010-vhrb-report.pdf. Accessed 16 Jun 2015

14. Pollard C, Savage V, Landrigan T, Hanbury A, Kerr D. Food Access and Cost Survey. Perth: Department of Health, WA; 2015.

15. Williams P. Monitoring the affordability of healthy eating: a case study of 10 years of the Illawarra Healthy Food Basket. Nutrients. 2010;2(11):1132-40.

16. Queensland Government. 2014 Healthy Food Access Basket Survey. 2015. http://www.health.qld.gov.au/research-reports/reports/food/access/ overview/default.asp. Accessed 8 Feb 2016.

17. National Health and Medical Research Council. A Modelling System to Inform the Revision of the Australian Guide To Healthy Eating. 2011. https:// www.eatforhealth.gov.au/sites/default/files/files/public consultation/n55a dietary_guidelines_food_modelling_111216.pdf. Accessed 11 Feb 2016

18. Hawkes C. Food taxes: what type of evidence is available to inform policy development? Nutr Bull. 2012;37(1):51-6.

19. WHO Europe. Using price policies to promote healthier diets WHO Regional Office for Europe UN City. Copenhagen: WHO Europe. 2015. http://www. euro.who.int/_data/assets/pdf file/0008/273662/Using-price-policies-topromote-healthier-diets.pdf?ua=1. Accessed 17 Jun 2015

20. Australian Government. Re:think; Better tax, better Australia. Discussion Paper. Canberra: The Treasury. 2015. http://bettertax.gov.au/publications/ discussion-paper/. Accessed Mar 152015

21. Australian Taxation Office. GST Food Search: Detailed food list, for business Canberra: ATO. 2014. https://expertsystems.ato.gov.au/scripts/net/ SearchableFoodList/scSearchableFoodList.aspx?PID=68\&ms=Businesses. Accessed 11 Feb 2016

22. Jones NR, Conklin Al, Suhrcke M, Monsivais P. The growing price gap between more and less healthy foods: analysis of a novel longitudinal UK dataset. PLoS One. 2014;9(10):e109343.

23. Veerman $J$, Cobiac LJ. Removing the GST exemption for fresh fruits and vegetables could cost lives. Med J Aust. 2013;199(8):534-5.

24. Sacks G, Veerman JL, Moodie M, Swinburn B. Traffic-light'nutrition labelling and 'junk-food'tax: a modelled comparison of cost-effectiveness for obesity prevention. Int J Obes. 2011;35(7):1001-9.

25. Australian Bureau of Statistics. Statistical Area Level 2 (SA2). Canberra: ABS. 2010. http://www.abs.gov.au/ausstats/abs@.nsf/Latestproducts/ 88F6A0EDEB8879C0CA257801000C64D9. Accessed 11 Feb 2016

26. Xyris Software (Australia) Pty Ltd. FoodWorks 7 Professional [computer program]. 7th ed. 2012.

27. National Health and Medical Research Council. Nutrient Reference Values for Australia and New Zealand, Including Recommended Dietary Intakes 2006. https://www.nhmrc.gov.au/ files nhmrc/publications/attachments/ n35.pdf. Accessed 11 Feb 2016

28. Queensland Government. Median total family income (a)(b) by statistical area level 4 (SA4) (c), Queensland, 2001, 2006 and 2011. Queensland Government: Brisbane; 2014. http://www.qgso.qld.gov.au/products/tables/ median-family-income-sa4/index.php. Accessed 2 July 2015.

29. Queensland Council of Social Services. Cost of Living Report: Can low income households afford a basic standard of living? Regional Report Issue 1. Brisbane: QCOSS. 2014. https://www.qcoss.org.au/sites/default/files/ 20141215 CoL_Report_Regional_FINAL.pdf. Accessed 16 Jun 2015

30. Australian Bureau of Statistics. Household income and income distribution Australia. Canberra: ABS. 2013. http://www.ausstats.abs.gov.au/ausstats/ subscriber.nsf/0/B0530ECF7A48B909CA257BC80016E4D3/\$File/65230_201112.pdf. Accessed 11 Feb 2016

31. Rao M, Afshin A, Singh G, Mozaffarian D. Do healthier foods and diet patterns cost more than less healthy options? A systematic review and meta-analysis. BMJ Open. 2013;3(12):e004277.

32. Waterlander WE, De Mul A, Schuit AJ, Seidell JC, Steenhuis $I H$. Research Perceptions on the use of pricing strategies to stimulate healthy eating among residents of deprived neighbourhoods: a focus group study. Int J Behav Nutr Phys Act. 2010;7(44). doi:10.1186/1479-5868-7-44. 
33. Carlson A, Frazão E. Are healthy foods really more expensive? It depends on how you measure the price. USDA-ERS Economic Information Bulletin No. 96, 2012. http://www.ers.usda.gov/publications/eib-economic-informationbulletin/eib96.aspx. Accessed 11 Feb 2016.

34. Australian Bureau of Statistics. Australian Dietary Guidelines Food Price Indexes. Canberra: ABS. 2016. http://www.abs.gov.au/ausstats/abs@.nsf/ Latestproducts/6401.0Feature\%20Article1Dec\%202015?opendocument\& tabname $=$ Summary\&prodno $=6401.0 \&$ issue $=$ Dec\%202015\&num $=\& v i e w=$ Accessed 13 February 2016

35. Ball K, Timperio A, Crawford D. Neighbourhood socioeconomic inequalities in food access and affordability. Health Place. 2009;15(2):578-85.

36. Lee A, Rainow S, Tregenza J, Tregenza L, Balmer L, Bryce S, Paddy M, Sheard J, Schomburgk D. Nutrition in remote Aboriginal communities: lessons from Mai Wiru and the Anangu Pitjantjatjara Yankunytjatjara Lands. ANZJPH. 2015 online doi: 10.1111/1753-6405.12419

37. Australian Bureau of Statistics. Australian Aboriginal and Torres Strait Islander Health Survey: Nutrition Results - Food and Nutrients, 2012-13. Canberra: ABS. 2015. http://www.abs.gov.au/AUSSTATS/abs@.nsf/ DetailsPage/4727.0.55.0052012-13?OpenDocument. Accessed 11 Feb 2016

38. Human Rights and Equal Opportunity Commission. Close the Gap: national Indigenous health equality targets. Outcomes from the National Indigenous Health Equality Summit. Canberra: Australian Human Rights Commission. 2008. https://www.humanrights.gov.au/publications/close-gap-nationalindigenous-health-equality-targets. Accessed 11 Feb 2016

39. Mhurchu CN, Eyles H, Schilling C, Yang Q, Kaye-Blake W, Genç M, et al. Food prices and consumer demand: differences across income levels and ethnic groups. PLoS One. 2013;8(10):e75934.

40. Nghiem N, Blakely T, Wilson N. Price Elasticities for Health Economic Modelling of Food Pricing Interventions in Australia and New Zealand: Department of Public Health. Wellington: University of Otago; 2011.

41. Green R, Cornelsen L, Dangour AD, Turner R, Shankar B, Mazzocchi M, Smith RD. the effect of rising food prices on food consumption: systematic review with meta-regression. BMJ. 2013;346:f3703. doi:10.1136/bmj.f3703.

42. Cancer Council WA DW. FOODcents: State of Western Australia. 2015. http://www.foodcentsprogram.com.au. Accessed 11 Feb 2016

43. Vidgen HA, Gallegos D. What is food literacy and does it influence what we eat: a study of Australian food experts. Brisbane: Queensland University of Technology; 2011. http://eprints.qut.edu.au/45902/.

44. Roberto CA, Swinburn B, Hawkes C, Huang TT. Costa SA, Ashe M, et al. Patchy progress on obesity prevention: emerging examples, entrenched barriers, and new thinking. The Lancet. 2015. http://www.thelancet.com/ journals/lancet/article/PIIS0140-6736\%2814\%2961744-X/fulltext. Accessed 11 Feb 2016.

45. Ball K, McNaughton SA, Le HN, Gold L, Mhurchu CN, Abbott G, et al. Influence of price discounts and skill-building strategies on purchase and consumption of healthy food and beverages: outcomes of the Supermarket Healthy Eating for Life randomized controlled trial. Am J Clin Nutr. 2015; 101(5):1055-64.

\section{Submit your next manuscript to BioMed Central and we will help you at every step:}

- We accept pre-submission inquiries

- Our selector tool helps you to find the most relevant journal

- We provide round the clock customer support

- Convenient online submission

- Thorough peer review

- Inclusion in PubMed and all major indexing services

- Maximum visibility for your research

Submit your manuscript at www.biomedcentral.com/submit

) Biomed Central 